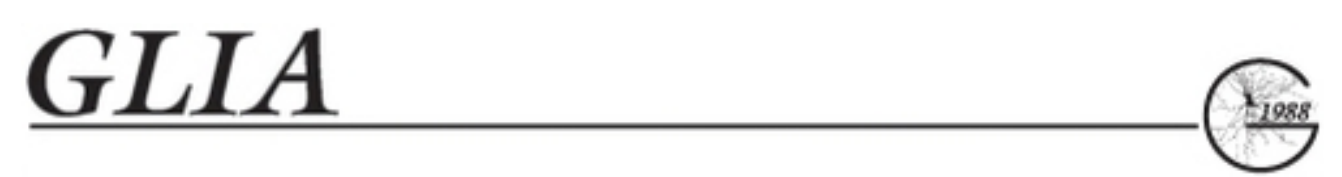

\title{
Astrocytes shape the plastic response of adult cortical neurons to vision loss
}

\begin{tabular}{|c|c|}
\hline Journal: & GLIA \\
\hline Manuscript ID & GLIA-00394-2019.R2 \\
\hline Wiley - Manuscript type: & Original Research Article \\
\hline $\begin{array}{r}\text { Date Submitted by the } \\
\text { Author: }\end{array}$ & 10-Mar-2020 \\
\hline Complete List of Authors: & $\begin{array}{l}\text { Hennes, Maroussia; KU Leuven, Biology } \\
\text { Lombaert, Nathalie; KU Leuven, Biology } \\
\text { Wahis, Jérôme; VIB KU Leuven Center for Brain \& Disease Research } \\
\text { Van den Haute, Chris; KU Leuven Biomedical Sciences Group } \\
\text { Holt, Matthew; VIB KU Leuven Center for Brain \& Disease Research } \\
\text { Arckens, Lutgarde; KU Leuven, Biology }\end{array}$ \\
\hline Topics: & Astrocytes, Glia and synaptic transmission, Ageing \\
\hline Techniques: & $\begin{array}{l}\text { Histological techniques, In situ hybridization, Neuroanatomical Methods, } \\
\text { Gene Cloning, Expression and Mutagenesis, Neurophysiology }\end{array}$ \\
\hline Key Words: & astrocytes, visual cortex, brain plasticity, adulthood \\
\hline
\end{tabular}

\section{SCHOLARONE Manuscripts}


1 Astrocytes shape the plastic response of adult cortical neurons to 2 vision loss

3 Maroussia Hennes ${ }^{1,2,3}$, Nathalie Lombaert ${ }^{1}$, Jérôme Wahis ${ }^{2,3,4}$, Chris Van den Haute ${ }^{5,6}$, 4 Matthew G Holt ${ }^{2,3,4}$, Lutgarde Arckens ${ }^{1,3}$

$5 \quad{ }^{1}$ KU Leuven, Department of Biology, 3000 Leuven, Belgium

$6 \quad{ }^{2}$ VIB KU Leuven Center for Brain \& Disease Research, 3000 Leuven, Belgium

$7 \quad{ }^{3}$ Leuven Brain Institute, 3000 Leuven, Belgium

$8{ }^{4}$ KU Leuven, Department of Neurosciences, 3000 Leuven, Belgium

$9{ }^{5}$ KU Leuven, Department of Biomedical Sciences, 3000 Leuven, Belgium

$10{ }^{6}$ KU Leuven Viral Vector Core, 3000 Leuven, Belgium

11 Running title: Astrocytes shape cortical plasticity

12 Corresponding author: Lutgarde Arckens, Laboratory of Neuroplasticity and Neuroproteomics, KU 13 Leuven, Naamsestraat 59, box 2467, B-3000 Leuven, Belgium, Telephone: +32 1632 39 51, E14 mail: Lut.arckens@kuleuven.be

15 Acknowledgements: We are grateful to Ria Vanlaer and Lieve Geenen for expert technical 16 assistance. This work was supported by the Fund for Scientific Research-Flanders to LA (Grant 17 number G061216N), to MGH (Grant numbers $1523014 \mathrm{~N}$ and $1527315 \mathrm{~N}$ ), to JW (Postdoctoral 18 fellowship, grant number $12 \mathrm{~V} 7519 \mathrm{~N}$ ), KU Leuven Research Council grant to LA (grant 19 C14/16/048), a Hercules Foundation Grant to LA (Grant AKUL-09-005), European Research 20 Council Starting Grant (281961) and VIB Institutional Funding to MGH. Table of contents image 21 was created with BioRender.com. The data that support the findings of this study are available 22 from the corresponding author upon reasonable request.

23 Main points:

- Visual deprivation induces a fast and persistent upregulation of Aldh1/1-positive astrocytes

- Acute fluorocitrate-induced silencing of astrocytes impedes neuronal reactivation

- $\mathrm{G}_{\mathrm{i}}$ but not $\mathrm{G}_{\mathrm{q}} \mathrm{GPCR}$ astrocyte activation promotes adult cortical plasticity

Word count by section: Abstract (243), Introduction (520), Materials and Methods (2732), Results (2192), Discussion (1946), References (2056), Figure legends (1170) 


\section{Abstract}

Astrocytes are vital for preserving correct brain functioning by continuously sustaining neuronal activity and survival. They are in contact with multiple synapses at once allowing the expansion of local synaptic events into activity changes in neuronal networks. Furthermore, cortical astrocytes integrate local sensory inputs and behavioral state. From an anatomical, molecular and functional perspective, astrocytes are thus ideal candidates to influence complex large-scale brain mechanisms such as plasticity. We collected evidence for the astrocytic potential for plasticity modulation, using the monocular enucleation (ME) mouse model of visual cortex plasticity. The impact of one-eyed vision involves the functional recruitment of the deprived visual cortex by the spared senses within a 7-week time frame, reflecting a substantial change in sensory information processing. In visually deprived cortex a swift upregulation in Aldh1/1-positive astrocyte density lasts until maximal functional recovery is reached. Transient metabolic silencing of visual cortex astrocytes at the time of ME induction, through intracranial fluorocitrate injections, reveals that astrocytes are required on site to achieve adequate long-term neuronal reactivation. In addition, chronic stimulation by $G_{i}$ but not $G_{q} G P C R$ activation of

\section{Keywords}

54 Astrocytes - Visual cortex - Brain plasticity - Adulthood 


\section{Introduction}

57

Astrocytes are one of the most abundant cell types in the mammalian cerebral cortex, where they are intermingling between the neurons in a tiled fashion (Bushong, 2002; Halassa, 2007). At the level of the tripartite synapse, astrocytes provide surrounding neurons with structural, metabolic and trophic support. Gliotransmission enables them to actively participate in synaptic transmission and to modulate neuronal function (Araque, 2014, 1999; Parpura, 2010). In addition, during development astrocytes possess synaptogenic capacities that remain latent in the adult brain (Christopherson, 2005; Singh, 2016; Stogsdill, 2017) (for review see (Baldwin, 2017)). Due to their specialized highly branched morphology, in cortex, protoplasmic astrocytes are able to contact multiple synapses at once, allowing them to exert local modulatory synaptic effects which can be expanded to influence coordinated neuronal circuit activity (Bushong, 2002; Halassa, 2007; Oberheim, 2009). Through lateral regulation of synapses, astrocytes can even serve as an information processing bridge between synapses with no direct neuronal connectivity (Covelo, 2016).

Astrocytes are thus ideally positioned and molecularly equipped candidates to mediate complex large-scale brain processes such as brain plasticity. This intrinsic ability of the central nervous system (CNS) allows it to structurally and functionally adapt to a continuously changing environment. CNS plasticity is crucial during early development and remains important throughout adult life, to facilitate learning, memory formation and even to functionally recover from traumatic events (Hensch, 2005; Hübener, 2014; Pascual-Leone, 2005).

However, in adulthood, the capacity for neural plasticity is more constrained making it more difficult for the adult brain to functionally recover from an injury or a neurological disorder. Discovering new strategies to reopen and modulate a window of heightened plasticity holds great promise for new therapeutic interventions for plasticity-related brain diseases. As neuronal circuit regulators, astrocytes present themselves as potential entry points for the fine-tuning of adult brain plasticity.

To test how and when astrocytes are involved in brain-region specific circuit adaptations in the context of neuronal plasticity, we performed three types of experiments in the monocular enucleation (ME) model of cortical plasticity. This mouse model of acquired 
87 one-eyed vision leads to an extensive neuronal reactivation of both binocular and 88 monocular territories within the deprived visual cortex contralateral to the lost eye, during 89 a 7-week recovery period (7WME). The functional recovery is established through the recruitment of the deprived visual cortex by the spared senses. Potentiation of sparedeye inputs mainly leads to a reactivation of the binocular zone in the first weeks post-ME,

92 whereas reactivation of the monocular zone in the visual cortex is somatosensory, whisker-driven, in nature (Nys, 2014; Van Brussel, 2011). Through immunohistochemical analysis of the pan astrocyte marker aldehyde dehydrogenase 1 family member L1 (Aldh1l1), we probed for astrocyte density changes in the visually deprived cortical areas. To test whether functional cortical astrocytes on site are necessary for adequate neuronal

97 recovery we induced acute metabolic silencing of astrocytes in the deprived visual cortex at the time of ME induction, through direct fluorocitrate injection. In parallel experiments, we chronically modulated astrocyte activity through a chemogenetics approach to interrogate if enhancing G-protein coupled receptor (GPCR) associated $G_{i}$ or $G_{q}$ signaling in cortical astrocytes can boost neuronal reactivation after ME. 


\section{Materials and Methods}

\section{Animals}

115

116

117

118

119

120

121

122

123

A total of $78 \mathrm{C} 57 \mathrm{BI} 6 / \mathrm{J}$ mice, obtained from Janvier Labs (Le Genest-St-Isle, France) were used for this study. All mice were housed under standard laboratory conditions under a 10/14-h dark/light cycle with food and water available ad libitum. All experiments were approved by the Ethical Research Committee of the KU Leuven and were in accordance with the European Communities Council Directive of 22 September 2010 (2010/63/EU) and with the Belgian legislation (KB of 29 May 2013). Every effort was made to reduce animal suffering and to reduce the number of animals used.

\section{Monocular enucleation and tissue processing}

Monocular enucleation (ME) was performed as previously described (Aerts, 2014). Briefly, P120 adult mice $(n=51)$ were sedated by an i.p. injection of a mixture of ketamine hydrochloride $(75 \mathrm{mg} / \mathrm{kg}$, Dechra Veterinary Products, Eurovet) and medetomidine hydrochloride (1 $\mathrm{mg} / \mathrm{kg}$ Orion Corporation, Janssen Animal Health). To prevent dehydration, eye ointment (Tobrex, Alcon) was applied on the left eye. The right eye was carefully removed and the orbit was filled with hemostatic cotton wool (Qualiphar, Bornem, Belgium) in case of bleeding. To prevent pain, analgesics were injected subcutaneously (Metacam, $2 \mathrm{mg} / \mathrm{kg}, 0.1 \mathrm{~mL}$ ) and atipamezol hydrochloride was administered to reverse the anaesthesia $(1 \mathrm{mg} / \mathrm{kg}$ i.p., Orion Corporation, Elanco Animal Health). Following enucleation, the mice were housed in their home cages under standard laboratory conditions for a 3-day to 7-week recovery period. At the end of the recovery period, mice $(n=18)$ used for immunohistochemical analysis were deeply sedated by a lethal injection of Dolethal (1 $\mathrm{ml} / 1.5 \mathrm{~kg}$, Vétoquinol S.A., Belgium) before being transcardially perfused with a $1 \%$ paraformaldehyde (PFA) solution in phosphatebuffered saline (PBS). Next, the brains were removed and post-fixed overnight in a $4 \%$ PFA solution in PBS. Coronal brain slices of $50 \mu \mathrm{m}$ were prepared on a Vibratome (HM $650 \mathrm{~V}$, Microm Microtech, France). For the analysis of zif268 expression levels through radioactive in situ hybridization, the mice $(n=33)$ were placed overnight in a dark room to reduce zif268 expression to basal levels. The next day they were placed in a highly-lit 
142 environment for 45 min to induce maximal sensory-driven zif268 mRNA expression. Next,

143 the mice were sacrificed through cervical dislocation after which the brains were carefully 144 removed and immediately frozen in 2-methylbutane (Merck, Overijse, Belgium) at a 145 temperature of $-40{ }^{\circ} \mathrm{C}$. All brains were stored at $-80^{\circ} \mathrm{C}$ until sectioning. Series of $25 \mu \mathrm{m}-$ 146 thick sections were prepared on a cryostat (HM 500 OM, Microm, Thermo Scientific, 147 Walldorf, Germany), mounted on $0.1 \%$ poly-L-Lysine-coated (Sigma-Aldrich) slides, and 148 stored at $-20^{\circ} \mathrm{C}$ until further processing.

\section{Aldh1l1 immunohistochemical analysis and quantification}

150 To assess the ME-effect on astrocyte density, the pan-astrocytic marker Aldehyde 151 Dehydrogenase-1 Family Member L1 (Aldh1l1) was used to visualize cortical astrocytes. Aldh1l1 diamino-benzidine (DAB) stainings were performed on $50 \mu \mathrm{m}$ free-floating sections. First, a heat-induced epitope retrieval (HIER) was performed at $80^{\circ} \mathrm{C}$ for $30 \mathrm{~min}$ in a $10 \mathrm{mM}$ sodium citrate buffer $(\mathrm{pH}=6)$. The sections were then pretreated with $\mathrm{H}_{2} \mathrm{O}_{2}$ (0,3 \% in Tris-buffered saline, TBS), rinsed in TBS and preincubated for 1 hour in blocking buffer (2 \% milk (GE Healthcare, UK), $0.5 \%$ Triton X-100 in PBS (1x)). Next, slices were incubated overnight at RT with rabbit anti-Aldh1l1 (1:200 in 5\% bovine serum albumin (BSA; Sigma aldrich), $0.5 \%$ Triton X-100 in PBS (1x)) after which detection was performed using biotinylated goat anti-rabbit IgG (1:500 in TBS, 30 min; Dako, Carpinteria, CA) and streptavidin horseradish peroxidase (1:500 in TBS, 1 hour; Dako). A glucose oxidase DAB-nickel method was used for detection, resulting in a black precipitate providing a higher contrast for cell counting compared to immunofluorescencebased stainings (Shu et al., 1998). In order to correctly delineate the different areas in the visual cortex as well as the cortical layers for Aldh1l1 astrocyte counting, adjacent sections were Nissl-stained (1\% Thionin, Fluka, Sigma-Aldrich) according to standard procedures. Pictures of the stained sections were obtained at 20X (NA: 0.8) with a light microscope (Zeiss Axio Imager Z1) equipped with an AxioCam MRm camera (1388 X 1040 pixels) using the software program ZEN (Zen Pro 2010, Carl Zeiss, Benelux).

170 For quantification of the astrocyte density, Aldh1l1 positive astrocytes were manually 171 counted using ImageJ (Fiji, US National institutes of Health). Position of interareal 
172 borders, delineations of monocular and binocular territory, and the cortical layers were 173 transferred between pictures from the adjacent Nissl sections to the Aldh1/1-stained 174 sections (Fig. 1b). Aldh1/1-positive astrocytes from the monocular and binocular visual 175 cortex were counted in vertical rows of cortical bins perpendicular to the pial surface and 176 spanning all cortical layers. Total number of counted cells was then normalized to the 177 total cortical bin size and represented as astrocytes $/ \mathrm{mm}^{2}$. To assess differences in layer 178 distribution of astrocytes, bins were assigned to cortical layers to then perform a 179 frequency distribution analysis using the coordinates of the counted cells in the binocular 180 181 and monocular bins. Per layer, counted astrocytes are represented as a percentage of the total number over all layers, to investigate whether density changes are layer-specific. Data were analyzed from six sections per animal and for all conditions 6 animals were used.

\section{Stereotactic surgery}

185 Mice were anesthetized through i.p. injection with a mixture of ketamine hydrochloride 186 (75 mg/kg; Dechra Veterinary Products; Eurovet) and medetomidine hydrochloride (1 $\mathrm{mg} / \mathrm{kg}$; Orion, Janssen Animal Health) before being stereotactically injected through a craniotomy into the primary visual cortex (V1) of the left hemisphere: $3.4 \mathrm{~mm}$ posterior to bregma, $2.5 \mathrm{~mm}$ lateral to the midline, at a depth of $400 \mu \mathrm{m}$ (viral vectors) or $500 \mu \mathrm{m}$ (fluorocitrate) from the cortical surface (Franklin, 2013). Viral vectors or fluorocitrate were delivered via a glass capillary (20 $\mu \mathrm{m}$ tip diameter) using a Nanoject II Auto-Nanoliter Injector (Drummond Scientific, Broomall, PA). A multiple injection approach per craniotomy was chosen to reach an optimal volume of transfected/affected cortex, based on a maximal distance of $300 \mu \mathrm{m}$ between injections along the A-P and $\mathrm{M}-\mathrm{L}$ axis of the brain. After each injection, the capillary was left in place for 1 min before being slowly retracted. Following wound suturing, anesthesia was reversed by i.p. injection of atipamezol hydrochloride (1 mg/kg; Orion; Elanco Animal Health, Antwerp, Belgium).

199 The fluorocitrate compound was used to induce an astrocytic metabolic silencing at ME onset. The fluorocitrate solution was prepared as previously described (Hayakawa, 2010). Briefly, $8 \mathrm{mg}$ of DL-fluorocitric acid barium salt (Merck, Darmstadt, Germany) was 
dissolved in $1 \mathrm{ml}$ of $0.1 \mathrm{M} \mathrm{HCl}$. Three drops of $0.1 \mathrm{M} \mathrm{Na}_{2} \mathrm{SO}_{4}$ were added to precipitate $\mathrm{Ba}^{2+}$. Next, $2 \mathrm{ml}$ of $0.1 \mathrm{M} \mathrm{Na}_{2} \mathrm{SO}_{4}$ was added before centrifuging the solution for $5 \mathrm{~min}$ at $1000 \mathrm{~g}$. The supernatant was further diluted with $0.9 \% \mathrm{NaCl}$ to obtain a final concentration of $1 \mathrm{nmol} / \mu \mathrm{l}(\mathrm{pH}=7.4)$.

Intracranial injection of fluorocitrate was performed in P120 mice as described above. A total of 4 injections per craniotomy were performed, each consisting of a volume of 345 $\mathrm{nL}$ (in steps of $69 \mathrm{~nL}$ every $20 \mathrm{~s}$ ). To validate specific metabolic silencing of astrocytes by fluorocitrate, mice were transcardially perfused one or three days $(n=6)$ after intracranial injection for analysis. For characterization of the effect of astrocyte elimination in the ME model, mice were enucleated immediately after fluorocitrate or saline injection and were sacrificed by cervical dislocation after 7 weeks for in situ hybridization with probes specific for zif268 ( $n=12)$ (Fig. 1a).

\section{Viral vector injections}

To evaluate if stimulating astrocyte activity could influence neuronal recovery after ME, a chemogenetic approach was implemented.

All used vectors (rAAV2/5-GFABCD1-hM3Dq-mCherry, rAAV2/5-GFABCD1-hM4DimCherry, rAAV2/5-GFABCD1-mCherry,) were produced at the Leuven Viral Vector Core as previously described (Van der Perren, 2011). Viral vectors were diluted to obtain a final genomic titer of $1 \times 10^{12}$ genome copies $/ \mathrm{ml}$. The sequence coding for the fluorescent mCherry protein was added to all constructs for detection purposes. The combination of the vector serotype and promoter sequence was chosen to reach appropriate expression sufficiency and specificity in astrocytes only. The third sham-control vector expressing only mCherry (no DREADD construct) was used to create the control condition for correct comparative analysis. Intracranial injections of the viral vectors were performed in P105 mice as described above. Here triple injections were performed, each containing a volume of $200 \mathrm{~nL}$ (in steps of $50 \mathrm{~nL}$ every $20 \mathrm{~s}$ ). To validate the astrocyte tropism of the vectors and the functionality of the DREADD constructs, a first set of mice $(n=15)$ were intracardially perfused two weeks after vector injections for immunofluorescent-based analysis. For studying the DREADD effect in the ME model, mice $(n=21)$ were enucleated 
232 two weeks after vector injection, at P120. All mice then received daily CNO (5 mg/kg; i.p.; 233 Tocris Bioscience, Bristol, UK) for 7 weeks (up to day 168) before being placed in darkness overnight, sacrificed and processed for zif268 in situ hybridization.

Immunofluorescence verification of fluorocitrate and viral vector specificity and functionality Validation of fluorocitrate-induced astrocyte-specific suppression, confirmation of viral vector-promoter-based astrocyte-specific targeting and DREADD-construct functionality was assessed using free-floating immunofluorescence. Upon transcardiac perfusion with $1 \%$ PFA in PBS, brains were dissected and postfixed overnight in 4 \% PFA in PBS at 4 ${ }^{\circ} \mathrm{C}$. Fifty $\mu \mathrm{m}$-thick coronal sections were obtained using a Vibratome (HM $650 \mathrm{~V}$, Microm Microtech, France). After washing with PBS, the sections were blocked for 60 min with normal goat serum (Merck Millipore, Overijse, Belgium) and overnight incubated at RT with the primary antibodies diluted in Tris-NaCl blocking buffer (TNB). The next day, slices were washed and then incubated for $2 \mathrm{~h}$ at RT with the secondary antibodies diluted in TNB buffer followed by a 15 min incubation with DAPI $(2 \mu \mathrm{L} / 100 \mathrm{~mL}$ PBS, Sigma-Aldrich, St. Louis, MO, 3260). Sections were then mounted on glass slides and coverslipped using Mowiol solution. For the Aldh1l1 immunofluorescent stainings, a HIER pretreatment and adapted buffers were used as described above. Images were taken at 20X (NA: 0.8) with a fluorescence microscope (Zeiss Axio Imager Z1) equipped with an AxioCam MRm camera (1388 × 1040 pixels) and Apotome1 using the software program Zen (Zen Pro 2012, Carl Zeiss, Benelux).

Table 1. Overview of primary and secondary antibodies used for immunohistochemistry.

\section{In situ hybridization}

High-throughput radioactive in situ hybridization (ISH) experiments for the immediate early gene (IEG) zif268 were performed to obtain an elaborate molecular overview of neuronal activity patterns across all cortical layers in the different areas of the visual 
262 cortex. The IEG zif268 has previously been described as a predominant neuronal activity 263 marker in the mammalian brain (Mataga, 2001). Furthermore previous studies have

264

265 266

267

268

269

270

271

272

273

274

275

276

277

278

279

280

281

282

283

284

285

286

287

288

289

290

291 shown that increased zif268 expression levels correspond with increased neuronal activation and can be used to assess alterations in cortical reactivation after ME (Lombaert, 2018; Nys, 2015; Scheyltjens, 2018; van Brussel, 2009; Van Brussel, 2011). In this study, the same zif268 readout was used to characterize the effect of astrocyte modulation on the neuronal recovery in the ME model.

All zif268 ISH experiments were performed on series of coronal brain sections between bregma levels $-1.5 \mathrm{~mm}$ and $-5 \mathrm{~mm}$ using a mouse-specific synthetic oligonucleotide probe (Eurogentec, Seraing, Belgium) with sequence 5'ccgttgctcagcagcatcatctcctccagtttggggtagttgtcc-3'. Each probe was 3'-end labeled with ${ }^{33}$ P-dATP using a terminal deoxynucleotidyl transferase (Invitrogen, Carlsbad, CA). MiniQuick Spin Oligo Columns (Roche Diagnostics, Vilvoorde, Belgium) were used to separate unincorporated nucleotides from the labeled probe. Cryostat sections were fixated, dehydrated and delipidated. The radioactively labeled probes were added to a hybridization cocktail ( $50 \%$ formamide, 4x standard saline sodium citrate (SSC) buffer, 1x Denhardt's solution, 10\% dextran sulfate, $100 \mu \mathrm{g} / \mathrm{ml}$ herring sperm DNA, $250 \mu \mathrm{g} / \mathrm{ml}$ tRNA, $60 \mathrm{mM}$ dithiothreitol, $1 \%$ $\mathrm{N}$-lauroyl sarcosine, and $20 \mathrm{mM} \mathrm{NaHPO}_{4}, \mathrm{pH} \mathrm{7.4)}$ and applied to the cryostat sections ( $10^{6} \mathrm{cpm} /$ section). After overnight incubation at $37^{\circ} \mathrm{C}$ in a humid chamber, the sections were rinsed in $1 \mathrm{x}$ standard SSC buffer at $42{ }^{\circ} \mathrm{C}$, dehydrated, air-dried and exposed to an autoradiographic film (Biomax MR; Kodak, Rochester, NY). After 7 days, the films were developed in EMS replacement for Kodak developer D-19 (Electron Microscopy Sciences, Hatfield) and fixed in Rapid fixer (llford; Hypam; Kodak). Autoradiographic images of adjacent sections were scanned at 1200 dpi (CanoScan 9000F Mark II; Canon; Tokyo; Japan) and all images were similarly adjusted for brightness and contrast in Adobe Photoshop CS5 (Adobe Systems, San Jose, CA). Corresponding pseudocolor representations were made using Image $J$ and represent a false color coding of the gray values ranging from black (0) to white (255); low gray values are represented in black/blue and high gray values in white/red. 


\section{Histology and delineation of visual areas based on Nissl patterns}

294 Following ISH, sections were Nissl counterstained (1 \% Thionin; Fluka, Sigma-Aldrich) according to standard procedures to visualize cortical layers and the boundaries between the different visual areas, to aid in the interpretation of the zif268 expression patterns. Images of the Nissl sections were obtained at 5X (NA: 0.16) using a light microscope (Zeis Axio Imager Z1) equipped with an AxioCam MRm camera (1388 1040 pixels) using the software program Zen (Zen Pro 2012, Carl Zeiss, Benelux). Immunofluorescent mCherry stainings were performed on post-fixed (4\% PFA in PBS) cryostat sections adjacent to the Nissl-stained ISH sections to confirm vector-mediated expression in V1. This staining as well as the image acquisition was performed as described above. The stereotactic mouse brain atlas was implemented to delineate the areal borders as described previously (Franklin, 2013; Nys, 2015; van Brussel, 2009). In all figures, the interareal borders are demarcated by black arrowheads. From lateral to medial five subregions are defined: the lateral extrastriate cortex (V2L), which is subdivided into a monocular (V2Lm) and a binocular (V2Lb) zone; the primary visual cortex (V1), which is subdivided into a monocular (V1m) and a binocular (V1b) zone; the medial extrastriate cortex (V2M) (Fig. 1b).

\section{Top view and line graph representations of cortical neuronal activity}

311 To evaluate the extent of the neuronal reactivation based on zif268 expression levels in 312 the different experimental paradigms, a quantification of the optical density (OD) values 313 across different visual areas was performed using a custom-made Matlab (Matlab R2016; 314 Mathworks) script as previously described (Nys, 2015; Vreysen, 2017). Top view representations of zif268 expression levels across the primary and secondary visual areas were created to obtain a spatial overview of the neuronal recovery in the different

317 conditions. Hereto, zif268 sections from bregma levels -2 until -4.5 (100 $\mu \mathrm{m}$ spread between sections) were loaded in the Matlab script. The region of interest in the left hemisphere was demarcated by selecting the top and bottom edge of the cortex and the

320 border between the supra-granular (II/III and IV) and the infragranular (V and VI) layers.

321 The region of interest between the rhinal fissure and the medial border of V2M was 
322 divided into 50 equal segments from lateral to medial to create two lattices, corresponding

323 to layers II-IV and layers V-VI (Fig. 1b,c). To adjust for background signal, a small

324 rectangular reference area was demarcated in the white matter. Per segment, the mean

325 OD value was calculated and normalized to the reference region and then projected onto

326 a horizontal planar projection. The horizontal projections per segment and per animal

327 were used to create the top view image.

328 Line graph representations of the neuronal reactivation pattern were created to obtain a 329 more detailed visualization of the averaged zif268 expression levels per visual cortex 330 area. Three sections near the injection site (bregma levels; -3.4, -3.5, -3.6) were selected 331 per animal for every condition and loaded into the Matlab script. The region of interest in 332 the left hemisphere was again delineated by selecting the top and bottom edge of the 333 cortex, as well as the border between the supra-granular and the infragranular layers. 334 The region of interest was divided into 27 (supra-granular layers) or 28 (infragranular 335 layers) equal segments from lateral to medial to create two lattices corresponding to 336 layers II-IV and layers V-VI (Fig.1c). Per segment the OD value was calculated and 337 normalized as described above for the top view representations. Results are presented 338 in subregion-specific bar graphs illustrating the neuronal activity patterns for the different analyzed conditions.

\section{Statistics}

341 Statistical analysis was performed using GraphPad Prism 5.01 (GraphPad Software, Inc).

342 All cell counting data and relative OD-values of zif268 sections are presented as mean \pm 343 SEM. Normal distribution and equal variance between groups was tested. A one-way 344 Anova with Bonferroni post hoc tests was used for pairwise comparisons of the Aldh1/1345 astrocyte cell counting experiments. For the analysis of the ISH data, a non-parametric 346 test (Mann-Whitney-U) was applied for pairwise comparison. For all used tests, a 347 probability level of $<0.05$ was accepted as statistically significant $\left({ }^{*} p<0.05,{ }^{* *} p<0.01\right.$, $348 * * * p<0.001)$. 
350

351

352

353

354

355

356

357

358

359

360

361

362

363

364

365

366

367

368

369

370

371

372

373

374

375

376

377

378

379

\section{Results}

Monocular enucleation impacts cortical astrocyte density in a time- and brain region-dependent manner

In order to study the astrocyte potential for cortical plasticity modulation, we first assessed if our visual deprivation paradigm affects astrocytes, and in which time frame they are responding to the loss of visual inputs. Transcriptome analysis previously revealed that the metabolic enzyme Aldh1l1 is highly specific for astrocytes and has a broader expression pattern than other commonly used markers such as Glial fibrillary acidic protein (GFAP) (Cahoy, 2008). We therefore applied immunohistochemical analysis of the expression of this pan astrocyte marker to investigate the effect of ME on visual cortex astrocytes from 3 days up to 7 weeks post ME. Aldh1/1-positive astrocytes are clearly detected across all cortical layers and display their typical star-shaped morphology (Fig. $2 a, b)$. We counted the immunopositive astrocytes separately for the monocular and the binocular subdivisions of the deprived visual cortex on sections of control (normal sighted), 3-day enucleated (3dME), 3-week enucleated (3wME) and 7-week enucleated (7WME) mice. In the monocular cortex, astrocyte density is already significantly increased at the $3 \mathrm{dME}$ time point compared to the control mice, indicating that astrocytes are responding early on to the visual deprivation. This upregulation of Aldh1/1-positive cortical astrocytes remains apparent for weeks, suggesting that this density increase is not transient but related to the entire cortical recovery period (Fig. 2c). A layer distribution analysis of the astrocytes from the monocular visual cortex revealed uniform astrocyte density changes across all cortical layers (Fig. 2d). A similar increase in Aldh1/1-positive astrocytes occurs in the binocular zone of the deprived visual cortex after 3 days, 3 weeks and 7 weeks ME compared to the control mice (Fig. 2e). However, astrocyte density at the $7 \mathrm{WME}$ time point is already decreased compared to the earlier 3dME time point, which suggests that, at least in the binocular zone, astrocyte density may return to basal levels over time (Fig 2e). Evaluation of the astrocyte layer distribution pattern shows a more pronounced impact on layer $V$ of the binocular cortex already at $3 \mathrm{dME}$, possibly at the expense of layer $\mathrm{VI}$ since the astrocyte percentage in layer $\mathrm{VI}$ of $3 \mathrm{dME}$ mice is decreased compared to the control condition. This altered distribution pattern of astrocytes may 
380 indeed suggest the occurrence of a relative astroglial redistribution across cortical layers

381 in the binocular zone.

382 Overall, the detailed analysis of the ME-effect on the spatial distribution of Aldh1/1383 positive astrocytes in visual cortex revealed a swift astrocyte response to the loss of visual 384 input that persists throughout all assessed time points, indicating that early, long-lasting 385 astrocyte contributions might be crucial to modulate the neuronal reactivation outcome of sensory-deprivation induced cortical plasticity. In addition, ME resulted in a redistribution of visual cortex astrocytes in favor of infragranular layer $\mathrm{V}$ in the binocular cortical territory.

Cortical fluorocitrate injection leads to a selective, reversible silencing of visual 389 cortex astrocytes

390 Considering that cortical astrocytes show an early response to the drop in neuronal 391 activity by upregulating their density swiftly, their initial presence might be necessary to 392 modulate the cortex towards long-term neuronal recovery. We hypothesized that by 393 experimentally blocking the early astrocyte response in V1, the plasticity-induced 394 neuronal reactivation would be hampered. To test this theory, fluorocitrate was intracranially injected into V1 in order to first assess in normal mice if this approach can locally metabolically silence visual cortex astrocytes. Figure 3 shows a clear drop in Aldh1/1-positive astrocytes at one day post-injection, localized to the fluorocitrate injection site (Fig. 3a, white rectangle). This is not resulting from tissue damage, since the nuclear marker 4',6-diamidino-2-phenylindole (DAPI) signal remains largely intact along all cortical layers (Fig. 3a). At one day post-injection, this metabolic suppression is highly selective for astrocytes since no apparent changes could be observed in the microglial (TMEM119) or neuronal (NeuN) cell density (Fig. 3b, Supplementary Fig. 1), in line with the DAPI findings. This fluorocitrate-induced astrocyte depression appears transient as 404 the effect is already largely reversed three days post-injection, when Aldh1/1-positive astrocytes are again detected at the injection site (Fig. 3c). The coincident induction of GFAP expression in cortical astrocytes is partially due to the intracranial surgery itself but seems to be more pronounced than in saline injected mice (Supplementary Fig.2). Most

408 likely, this reawakening of visual cortex astrocytes is accompanied by a compensatory 409 reaction leading to a heightened GFAP expression. 
410

411

412

413

414

415

416

417

418

419

420

421

422

423

424

425

426

427

428

429

430

431

432

433

434

435

436

437

438

439

Early transient silencing of astrocytes impairs functional recovery of the deprived visual cortex

As astrocytes are capable of modulating neuronal activity, their early onset rise in density after ME might be necessary for the plastic neuronal recovery of the deprived visual cortex. To assess to what degree the early astrocytic response is contributing to the neuronal reactivation, we next injected fluorocitrate intracranially into the deprived visual cortex moments before performing ME (Fig. 1a). Mice were left to recover for 7 weeks in their home cages, a period in which ME-induced plasticity normally leads to extensive recovery of neuronal activity in the visual cortex. To compensate for possible effects of the stereotactic surgery, we injected sham control ME mice with the same volume of saline for comparison. Immediate early gene zif268 expression levels were used as the readout for experience-induced neuronal activity and consequently the extent of plasticitymediated recovery from ME. Considering that ME differently affects neuronal activity across cortical layers and taking into account the heterogeneity of cortical astrocytes, supra-granular (II/III and IV) and infragranular (V and VI) layer activity were separately evaluated (Bayraktar, 2019; Clarke, 2018; Xin, 2018; Zhang, 2010).

The creation of top view representations of the zif268 expression pattern and levels across visual areas reveals differences in the spatial extent of the neuronal reactivation between the fluorocitrate and saline injected ME mice (Fig. 4a). The fluorocitrate condition shows an overall smaller spread in zif268 expression in both the supra- and the infragranular layers (Fig. 4a, left and right panels). This lowered neuronal reactivation is most pronounced for the monocular visual cortex, indicating that the typical features of neuronal recovery (Fig. 1d), namely binocular zone expansion and monocular region reactivation, are hampered (Fig. 4a). In order to visualize the impact on neuronal reactivation in more detail, we also created line graph representations reflecting the neuronal activity levels of brain sections surrounding the injection site (bregma: -3.4). Figure $4 \mathrm{~b}$ contains representative images of such sections processed for zif268 mRNA epxression, with their corresponding pseudocolor images, with black arrowheads indicating the boundaries between the analyzed visual cortical areas. Similar coronal sections from the group of mice from both conditions $(n=6)$ were used to create the line 
graphs representing the neuronal activity pattern based on averaged zif268 expression levels across these anatomically delineated visual areas (dotted vertical lines represent interareal borders and correspond to the black arrowheads in figure $4 \mathrm{~b})$. The supragranular layers (left line graph) clearly display lower zif268 expression levels in the fluorocitrate condition (blue) compared to the saline condition (black) illustrating a reduced neuronal reactivation, similar to the observation made in the supra-granular top view. For the infragranular layers, the line graph (Fig. 4c, right graph) shows a complete overlap between the fluorocitrate (blue) and saline (black) condition, indicative of similar averaged zif268 expression. Area-specific statistical analysis further revealed that the averaged zif268 expression in the fluorocitrate condition is significantly lower in the supragranular layers of $\mathrm{V} 1$ and the monocular region of the visual cortex, including $\mathrm{V} 1 \mathrm{~m}$ and V2M, compared to saline control. No significant differences could be observed in the supra-granular layers of the binocular zone (V2Lb and V1b) or in the infragranular layers (Fig. 4d).

These results show that an early and transient silencing of astrocytes in visual cortex impacts the neuronal reactivation process specifically in the supra-granular layers of the deprived visual cortex, thereby revealing that astrocytes are indispensable for plasticity following ME. The effect of fluorocitrate-induced astrocyte silencing is mostly concentrated in monocular visual cortex, where the loss of visual input due to ME is the greatest. This indicates that exactly where neuronal activity drops, due to an instant and complete loss of sensory input, a fast response of astrocytes is crucial for adequate functional recovery in the longer term.

Astrocyte specific targeting of AAV2.5-GFABCD1D vector and validation of the functionality of DREADD constructs

Astrocytes have been shown to be essential for synaptogenesis and subsequent modulation of synaptic function. We hypothesized that modulating their activity might therefore lead to a boost in neuronal recovery. To be able to chronically steer solely the activity of astrocytes, we implemented a chemogenetics approach. Via a recombinant adeno-associated viral vector approach, we first expressed hM3Dq and hM4Di DREADD constructs in normal visual cortex astrocytes. We obtained specific astrocyte expression, 
470 by using an AAV2.5 viral vector with a modified GFABCD1D promotor and mCherry 471 reporter gene. To validate the astrocyte targeting we performed immunofluorescent 472 double stainings. Figure 5a shows the clear co-labeling between the mCherry reporter 473 gene and astrocyte specific cytoplasmic (Aldh111, GFAP) as well as plasma membrane 474 markers (Connexin43, Kir4.1) in visual cortex. The used viral vector construct shows no 475 co-labelling with any of the other neuron (NeuN), interneuron (Parvalbumin), microglia (TMEM119) or oligodendrocyte (Olig2) cell-specific markers (Fig. 5b). Upon acute i.p. injection of CNO, the immediate early gene Fos is expressed only in hM3Dq- and hM4Diexpressing visual cortex astrocytes but not in astrocytes transduced with the mCherry control vector (Fig. 5c).

Chronic $G_{i}$ but not $G_{q}$ GPCR activation of astrocytes enhances neuronal plasticity in the ME model

482 In order to show that astrocytes are not only necessary for the occurrence of neuronal reactivation after ME but that they are capable of boosting the recovery process, we next applied the validated DREADD toolbox to reliably enhance their activity in the ME model. As our results indicated that astrocytes are fast responders to visual deprivation and that their increased detection lasts until the final phase of the plastic recovery, we opted to modulate their activity long-term until the end of the model. Therefore, mice of all conditions (mCherry, hM3Dq, hM4Di) received daily i.p. CNO $(5 \mathrm{mg} / \mathrm{kg})$ injections for 7 weeks following ME. The zif268 mRNA-based readout was again used to assess the neuronal reactivation pattern separately for layers II-IV and V-VI in the different areas of 491 the deprived visual cortex.

Comparison of the top view representations of the spatial zif268 expression patterns reveals a more confined expression pattern in the hM3Dq injected mice, and a broader expression pattern in the hM4Di injected mice compared to the mCherry controls. Again, a more pronounced impact is observed for the supra-granular layers of the visual cortex (Fig. 6a). The spatial zif268 expression pattern of the hM4Di condition spreads further into the monocular territory of the visual cortex indicating that chronically enhancing $G_{i}$ signaling in astrocytes can lead to a more pronounced reactivation of the monocular cortex (Fig. 1d). On the other hand, the spatial reactivation pattern of the hM3Dq 
500 conditions seems to be more confined than what is seen in mCherry control mice, 501 suggesting that chronic $\mathrm{G}_{\mathrm{q}}$ signaling in astrocytes can hamper the spread of neuronal 502 activity across the visual cortex. Representative images of zif268 mRNA labeled sections 503 from hM3Dq, mCherry and hM4Di expressing mice and their corresponding pseudocolor 504 images, with black arrowheads representing the boundaries between the analyzed visual areas, are shown in figure 6b. Similar to the top view observation, the hM4Di condition shows higher zif268 expression in V1 compared to the mCherry control. Figure $6 \mathrm{~b}$ also illustrates correct vector localization in V1 based on mCherry reporter gene expression for the three experimental conditions. Line graph representations based on such sections surrounding the injection site (bregma: -3.4) were again created to obtain a more detailed overview of the neuronal reactivation pattern across visual areas. The supra-granular layers (left line graph) clearly show an overall lower averaged zif268 expression in the hM3Dq condition (green) compared to the mCherry control (black) across the analyzed visual areas. Yet compared to the mCherry control (black), the hM4Di (red) condition is characterized by increased averaged zif268 expression levels for the supra-granular layers in V1 as well as the monocular cortex. The infragranular layers display similar averaged zif268 expression levels across all conditions (Fig. 6c). Statistical analysis of the relative zif268 expression levels in the different visual areas revealed that the hM3Dq condition (green) is significantly less reactivated in the supra-granular layers of the binocular zone compared to the mCherry control (black). This suggests that $\mathrm{G}_{\mathrm{q}}$ signaling hampers neuronal recovery by decreasing binocular zone expansion and monocular zone reactivation. hM4Di zif268 (red) expression levels are significantly upregulated in the supra-granular layers of the monocular region of $\mathrm{V} 1(\mathrm{~V} 1 \mathrm{~m})$ compared to the mCherry control (black). As such, modulation of astrocyte activity by enhancing $\mathrm{G}_{\mathrm{i}}$ signaling is thus capable of boosting neuronal reactivation in the monocular zone of the deprived visual cortex. Comparison of hM3Dq and hM4Di averaged zif268 expression levels reveals that 526 the hM4Di condition is showing significantly higher neuronal activity levels in the supra527 granular layers of the binocular and the monocular zone as well as in V1 (Fig. 6d).

528 In conclusion, besides being required on site for adequate neuronal reactivation to occur 529 in deprived cortical territory, astrocyte stimulation even holds the potential to boost the 
530 neuronal plasticity process, which further highlights their capacity as neuronal modulators 531 and plasticity mediators. 
533

534

535

536

537

538

539

540

541

542

543

544

545

546

547

548

549

550

551

552

553

554

555

556

557

558

559

560

561

562

563

\section{Discussion}

Our results demonstrate how protoplasmic astrocytes actively contribute to large-scale reorganization of adult cortical function induced by sensory deprivation. The astrocyte density quickly increased in response to an instant drop in neuronal activity caused by loss of sensory inputs throughout the relevant cortical territories. Such changes in astrocyte density in the context of plasticity paradigms have already been described. Saur et al. reported how physical exercise, capable of positively affecting neuroplasticity, leads to a coincident increase in astrocyte density in the CA1 rat hippocampal region (Saur, 2014). On the other hand, a decrease in hippocampal astrocyte quantity has been identified in neurological disorders associated with disturbed neuroplasticity such as major depression disorder (Cantone, 2017; Cobb, 2016). Specifically, for the visual system Gonzalez et al. could show that ME leads to a long-term upregulation of astrocyte numbers in the rat lateral geniculate nucleus. They suggested that in subcortical visual nuclei such changes in astrocyte density (in response to ME) occur initially to facilitate removal of neuronal debris, followed at later points by synaptic reorganization (Gonzalez, 2006). We showed here that at the level of the visual cortex, which is not a direct retinal target region, a similar rise in the number of astrocytes already occurs in the first few days post injury. As no neuronal degeneration or reactive GFAP-positive astrocytes are detected in the visual cortex after ME (Nys, 2014; Supplementary Fig.3), this early cortical astrocyte response seems evoked by the acute drop in neuronal activity per se.

Besides alterations in astrocyte density, also shifts in astrocyte subpopulations have been observed in response to visual deprivation (Cornett, 2011). Our findings may support the possibility that normally active mouse visual cortex contains a silent, Aldh1/1-negative astrocytic subpopulation that gets triggered upon ME. However, as Aldh1/1 is a relatively 'new' astrocytic marker, more research is needed to reveal if Aldh1l1-negative astrocytes exist in mouse cortex under normal physiological conditions.

The astrocytic response to ME appeared heterogeneous throughout the mouse visual cortex. In the monocular territory, the rise in Aldh1l1 positive astrocytes occurred uniformly across all cortical layers and lasted the whole 7-week recovery period. In the binocular zone, the upregulation occurred with the same swiftness but started to downregulate towards the end of the model, when maximal functional recovery is 
564

565

566

567

568

569

570

571

572

573

574

575

576

577

578

579

580

581

582

583

584

585

586

587

588

589

590

591

592

593

established (Van Brussel, 2011). Here, the increase in density was also more pronounced in the infragranular layers, especially in layer $\mathrm{V}$ of the visual cortex. These subregionspecific differences correlate with previous research from our lab showing that the impact of ME on neuronal activity in the monocular region is visible throughout all layers of the cortex, whereas in the binocular region the infragranular layers show the greatest loss (Van Brussel, 2011). These results thus indicate that astrocytes sense the region-specific loss of visual input and compensate accordingly. Neuronal activity has been shown before to affect and shape the surrounding astrocytic population. By altering the astrocytic transcriptome, neuronal activity can modify the astrocytes' functional, molecular and anatomical state (Farmer, 2016; Hasel, 2017). Furthermore, as visual stimulation elicits a response in mouse cortical astrocytes that can be influenced by the animal's behavioral state (Slezak, 2018; Sonoda, 2018), it is not surprising that also the loss of visual input would elicit a profound astrocytic response. As astrocytes are known to influence neuronal activity and synaptic transmission, specific alterations in astrocyte presence and functionality are most likely required for promoting brain region-specific synaptic rearrangement and reestablishment of neuronal functions in the visual cortex. Possibly, a reinstatement of latent synaptogenic abilities in astrocytes in the adult brain might induce excitatory synaptogenesis promoting functional cortical recovery (Baldwin, 2017; Singh, 2016).

Our prediction was that blocking the initial astrocyte response to the sudden loss of sensory input would lead to impairment of neuronal recovery long-term in the deprived cortex. Acute fluorocitrate-induced metabolic suppression was clearly successful as we hardly detected any Aldh1/1-positive astrocytes in the immediate vicinity of the injection site at 1 day post-injection, an effect which had reversed at 3 days, comparable to previous studies on acute fluorocitrate exposure (Paulsen, 1987; Ronald Zielke, 2007; Zielke, 2009). We also demonstrated that fluorocitrate did not have a parallel overt effect on the relative amount or shape of the surrounding neuronal or microglial cell populations, consistent with previous findings showing activation of microglial cells related to astrocytic stress only following chronic fluorocitrate treatment (Kuter, 2018). 
594 Furthermore, our results clearly indicate that when such an acute and selective metabolic 595 silencing of astrocytes is applied to the deprived visual cortex of ME mice it is capable of impeding long-term neuronal recovery, confirming that astrocytes are responsive at the time of ME induction and act as an integral component in the plasticity process underlying the regain of cortical functionality.

Gliotransmission enables astrocyte-neuron communication at the level of the tripartite synapse. Astrocytes have the capacity to release a wide variety of molecules responsible for maintaining neuronal function and survival. Cortical astrocytes can, for instance, enhance excitatory synaptic transmission by releasing gliotransmitters such as glutamate and D-serine (Fossat, 2011; Navarrete, 2012). In addition, they supply surrounding neurons with the necessary energy and metabolic substrates for neuronal biosynthesis (Weber, 2015). Thus, by incapacitating astrocytes at ME onset, neurons will be stripped of critical building blocks to regain their function.

The initial brain state, determined by the excitation-inhibition balance, at the time of deprivation, can have long-lasting effects on functional recovery. Enhancement of inhibitory neurotransmission prior to $\mathrm{ME}$, through either dark exposure or optogenetic activation of somatostatin interneurons, results in reduced functional recovery of the monocular region of the deprived visual cortex (Nys, 2015; Scheyltjens, 2018). Interfering with the natural astrocytic response to ME results in a similar phenotype. Such an early astrocyte response appears to be a necessary step in establishing a plastic state that allows full recovery of neuronal activity.

The DREADD technique allows time-dependent activation of visual cortex astrocytes (Armbruster, 2007). Durkee et al. recently revealed that both $G_{q} G P C R$ and $G_{i} G P C R$ activation leads to the cellular activation of astrocytes. Furthermore they showed, in acute hippocampal slices, that astrocyte activation through $G_{q}$ and $G_{i}$ signaling induces glutamate release, able to enhance neuronal excitability (Durkee, 2019). In our hands, acute $\mathrm{CNO}$ administration also activated both $\mathrm{G}_{\mathrm{q}^{-}}(\mathrm{hM} 3 \mathrm{Dq})$ and $\mathrm{G}_{\mathrm{i}^{-}}(\mathrm{hM} 4 \mathrm{Di})$ expressing astrocytes in the visual cortex, based on CNO-driven Fos expression in DREADD transduced astrocytes. In the ME model, long-term CNO-based cortical astrocyte activation through $\mathrm{G}_{\mathrm{q}}$ GPCR activation resulted in a diminished local neuronal reactivation 
625 compared to the control condition. $\mathrm{G}_{\mathrm{q}}$ GPCR activation of hippocampal astrocytes 626 induces the release of glutamate and D-serine which activates neuronal NMDA receptors 627 leading to a heightened neuronal excitability (Adamsky, 2018; Durkee, 2019). We 628 therefore expected that enhancing $G_{q}$ signaling in cortical astrocytes would lead to a 629 faster functional recovery of the deprived neurons. However, we must take into account the fact that the astrocyte population is highly heterogeneous between brain areas.

631 Different subtypes of astrocytes exist across different areas all showing distinct functional, 632 morphological and molecular profiles (Batiuk, 2019; Bayraktar, 2019; Clarke, 2018; Xin, 2018; Zhang, 2010). By activating $G_{q}$ GPCR in visual cortex astrocytes we are thus potentially manipulating a molecularly different type of cell, leading to different effects on neuronal function compared to hippocampus. Furthermore, in our case, the $G_{q}$ GPCR activated astrocytes find themselves in an environment of altered, sensory-deprived neurons which could further diversify their molecular composition compared to normal physiological conditions. In a model of addiction, glutamate release through $\mathrm{G}_{\mathrm{q}} \mathrm{GPCR}$ activation of astrocytes is, for instance, directed to the pre-synaptic mGluR2/3 receptor that, upon activation, prevents synaptic neurotransmitter release (Scofield, 2015). Likewise, visual deprivation also modifies the molecular architecture of synapses, by affecting glutamate release probability and composition of NMDA receptors, leading to enhanced NMDA-signaling, specifically in the supra-granular layers of the visual cortex (Yashiro, 2005). Glutamate, potentially released through $\mathrm{G}_{\mathrm{q}}$ activation of astrocytes in the ME model, might thus be acting on glutamate receptors that block presynaptic release, 646 such as mGluR2/3, leading to lowered neuronal excitability in the supra-granular layers 647 of the visual cortex.

On the other hand, $\mathrm{G}_{i}$ GPCR activation of visual cortex astrocytes in the ME model had a 650 positive influence on the plasticity process, generating a boost in neuronal reactivation in comparison to what happens in control or $\mathrm{G}_{\mathrm{q}}$ DREADD ME mice. Brief stimulation of cortical astrocytes with GABA, which also induces $G_{i}$ GPCR signaling, leads to longlasting calcium oscillations in astrocytes, able to enhance synchronization of activity 654 between pyramidal neurons (Mariotti, 2016). Stimulating plasticity with $\mathrm{G}_{\mathrm{i}} \mathrm{GPCR}_{\mathrm{C}}$ astrocyte 655 activation might thus have a longer lasting impact on surrounding neurons, which 
656 confirms the potential of this pathway to positively influence long-lasting plasticity

657

658

659

660

661

662

663

664

665

666

667

668

669

670

671

672

673

674

675

676

677

678

679

680

681

682

683

684

685

686 processes.

More recently, RNAseq of $G_{i}$ GPCR activated striatal astrocytes revealed that stimulating this signaling pathway leads to the activation of thrombospondin 1 (TSP1), a latent synaptogenic cue in adult mice, and also important for intracortical synapse formation. As a result, this induced an increase in excitatory synapse formation, synaptic function and neuronal firing in mouse striatum (Nagai, 2019). Astrocyte-induced synaptogenesis is predominantly present in the developing mouse brain. Through the secretion of synaptogenic factors, such as TSP1, Hevin and TGF-B1, that interact with specific neuronal receptors and adhesion molecules, astrocytes are involved in the formation and maturation of excitatory synapses (Baldwin, 2017; Singh, 2016; Stogsdill, 2017). In the adult brain, astrocytic expression of some of these factors is reduced, making mature astrocytes less synaptogenic (Christopherson, 2005). However, after brain injury or specific activation, such as with the $G_{i}$ DREADD, mature astrocytes may reinstate their immature synaptogenic state to aid in neuronal recovery (Liauw, 2008; Nagai, 2019; Teng-nan, 2003). By stimulating $G_{i}$ GPCR signaling in visual cortex astrocytes during the plastic recovery upon ME, we might thus have induced a reversal of local astrocytes into a more immature phenotype capable of inducing new excitatory synapse formation, responsible for the observed boost in neuronal reactivation. This interpretation appears in line with previous research on adult cat visual cortex showing that transplantation of immature astrocytes reintroduces critical period-like ocular dominance plasticity (Müller, 1989). More recently, Blanco-Suarez et al. showed that manipulating astrocytic synapsemodifying signals, by knocking out the chordin like 1 gene, is also capable of boosting visual cortex plasticity (Blanco-Suarez, 2018).

Functional manipulation of astrocytes in the ME model seems to mainly affect the supragranular layers of the deprived visual cortex, even though infragranular astrocytes are clearly also transduced. These results thus suggest that upper layer astrocytes are more capable of affecting the plasticity outcome. Previous research on astrocyte heterogeneity in the cortex also states that upper layer astrocytes are morphologically and molecularly 
687 better equipped to modify excitatory synapse transmission and formation (Bayraktar, 688 2019; Lanjakornsiripan, 2018).

689

690 Finally, our observations of astrocyte contributions to experience-dependent brain 691 plasticity may be extrapolated to cortical reorganization processes observed after stroke 692 or traumatic brain injury, as in these cases astrocyte-mediated synaptic plasticity also 693 underlies functional recovery (Kim, 2018; Nikolakopoulou, 2016). Metabolic inhibition of 694 astrocytes, as in our study, worsens the behavioral outcome in a mouse model of stroke 695 (Hayakawa, 2010). Therefore, we propose $G_{i}$ GPCR activation of astrocytes as a potential 696 therapeutic strategy to promote recovery of neuronal function not only after sensory 697 deprivation but also after direct cortical injury.

698

699 To conclude, our results show that astrocytes are central players in the plasticity-induced 700 functional reactivation of sensory-deprived visual cortex in the ME model. Furthermore, 701 stimulation of $G_{i}$ signaling in cortical astrocytes actually boosted the neuronal reactivation 702 process, possibly by reverting astrocyte to a more immature and synaptogenic state. We 703 have, therefore, identified $G_{i}$ GCPR activation of astrocytes as an interesting therapeutic 704 pathway to reopen plasticity windows in the adult mammalian cortex. Furthermore, this 705 study adds to the growing knowledge on astrocytes as neuronal regulators and mediators 706 of cortical plasticity. 


\section{References}

Adamsky, A., Kol, A., Kreisel, T., Doron, A., Ozeri-Engelhard, N., Melcer, T., ... Goshen, I. (2018). Astrocytic Activation Generates De Novo Neuronal Potentiation and Memory Enhancement. Cell, 174(1), 59-71.e14. https://doi.org/https://doi.org/10.1016/j.cell.2018.05.002

Aerts, J., Nys, J., \& Arckens, L. (2014). A Highly Reproducible and Straightforward Method to Perform <em>ln Vivo</em> Ocular Enucleation in the Mouse after Eye Opening. Journal of Visualized Experiments. https://doi.org/10.3791/51936

Araque, A., Carmignoto, G., Haydon, P. G., Oliet, S. H. R., Robitaille, R., \& Volterra, A. (2014). Gliotransmitters Travel in Time and Space. Neuron, 81(4), 728-739. https://doi.org/https://doi.org/10.1016/j.neuron.2014.02.007

Araque, A., Parpura, V., Sanzgiri, R. P., \& Haydon, P. G. (1999). Tripartite synapses: glia, the unacknowledged partner. Trends in Neurosciences, 22(5), 208-215. https://doi.org/https://doi.org/10.1016/\$0166-2236(98)01349-6

Armbruster, B. N., Li, X., Pausch, M. H., Herlitze, S., \& Roth, B. L. (2007). Evolving the lock to fit the key to create a family of $\mathrm{G}$ protein-coupled receptors potently activated by an inert ligand. Proceedings of the National Academy of Sciences, 104(12), 5163 LP - 5168. https://doi.org/10.1073/pnas.0700293104

Baldwin, K. T., \& Eroglu, C. (2017). Molecular mechanisms of astrocyte-induced synaptogenesis. Current Opinion in Neurobiology, 45, 113-120. https://doi.org/https://doi.org/10.1016/j.conb.2017.05.006

Batiuk, M. Y., Martirosyan, A., Wahis, J., de Vin, F., Marneffe, C., Kusserow, C., ... Holt, M. G. (2019). Unraveling region-specific astrocyte subtypes at single cell resolution. Nature Communications.

Bayraktar, O. A. B. T. H. S. K. V. M. M. P. D. B. H. L. Y. A. M. H. P. K. B. A. R. K. P. M. F. K. R. S. J. S. K. C. S. E. H. E. (2019). Single-cell in situ transcriptomic map of astrocyte cortical layer diversity. Nature Neuroscience, Accepted. 
Blanco-Suarez, E., Liu, T.-F., Kopelevich, A., \& Allen, N. J. (2018). Astrocyte-Secreted Chordin-like 1 Drives Synapse Maturation and Limits Plasticity by Increasing Synaptic GluA2 AMPA Receptors. Neuron, 100(5), 1116-1132.e13. https://doi.org/https://doi.org/10.1016/j.neuron.2018.09.043

Bushong, E. A., Martone, M. E., Jones, Y. Z., \& Ellisman, M. H. (2002). Protoplasmic Astrocytes in CA1 Stratum Radiatum Occupy Separate Anatomical Domains. The Journal of Neuroscience, 22(1), 183 LP - 192. https://doi.org/10.1523/JNEUROSCI.22-01-00183.2002

Cahoy, J. D., Emery, B., Kaushal, A., Foo, L. C., Zamanian, J. L., Christopherson, K. S., ... Barres, B. A. (2008). A Transcriptome Database for Astrocytes, Neurons, and Oligodendrocytes: A New Resource for Understanding Brain Development and Function. The Journal of Neuroscience, 28(1), 264 LP - 278. https://doi.org/10.1523/JNEUROSCI.4178-07.2008

Cantone, M., Bramanti, A., Lanza, G., Pennisi, M., Bramanti, P., Pennisi, G., \& Bella, R. (2017). Cortical Plasticity in Depression. ASN Neuro, 9(3), 17590914177115121759091417711512. https://doi.org/10.1177/1759091417711512

Christopherson, K. S., Ullian, E. M., Stokes, C. C. A., Mullowney, C. E., Hell, J. W., Agah, A., ... Barres, B. A. (2005). Thrombospondins Are Astrocyte-Secreted Proteins that Promote CNS Synaptogenesis. Cell, 120(3), 421-433. https://doi.org/https://doi.org/10.1016/j.cell.2004.12.020

Clarke, L. E., Liddelow, S. A., Chakraborty, C., Münch, A. E., Heiman, M., \& Barres, B. A. (2018). Normal aging induces A1-like astrocyte reactivity. Proceedings of the National Academy of Sciences, 115(8), E1896 LP-E1905. https://doi.org/10.1073/pnas.1800165115

Cobb, J. A., O'Neill, K., Milner, J., Mahajan, G. J., Lawrence, T. J., May, W. L., ... Stockmeier, C. A. (2016). Density of GFAP-immunoreactive astrocytes is decreased in left hippocampi in major depressive disorder. Neuroscience, 316, 209-220. https://doi.org/10.1016/j.neuroscience.2015.12.044 
763

764

765

766

767

768

769

770

771

772

773

774

775

776

777

778

779

780

781

782

783

784

785

786

787

788

789

790

Cornett, A., Sucic, J. F., Hillsburg, D., Cyr, L., Johnson, C., Polanco, A., ... Jarvinen, M. K. (2011). Altered glial gene expression, density, and architecture in the visual cortex upon retinal degeneration. Brain Research, 1422, 46-56.

https://doi.org/https://doi.org/10.1016/j.brainres.2011.09.011

Covelo, A., \& Araque, A. (2016). Lateral regulation of synaptic transmission by astrocytes. Neuroscience, 323, 62-66. https://doi.org/https://doi.org/10.1016/j.neuroscience.2015.02.036

Durkee, C. A., Covelo, A., Lines, J., Kofuji, P., Aguilar, J., \& Araque, A. (2019). Gi/o protein-coupled receptors inhibit neurons but activate astrocytes and stimulate gliotransmission. Glia, 67(6), 1076-1093. https://doi.org/10.1002/glia.23589

Farmer, W. T., Abrahamsson, T., Chierzi, S., Lui, C., Zaelzer, C., Jones, E. V, ... Murai, K. K. (2016). Neurons diversify astrocytes in the adult brain through sonic hedgehog signaling. Science, 351(6275), 849 LP - 854. https://doi.org/10.1126/science.aab3103

Fossat, P., Turpin, F. R., Sacchi, S., Dulong, J., Shi, T., Rivet, J.-M., ... Mothet, J.-P. (2011). Glial D-Serine Gates NMDA Receptors at Excitatory Synapses in Prefrontal Cortex. Cerebral Cortex, 22(3), 595-606. https://doi.org/10.1093/cercor/bhr130

Franklin, K. B. J., \& Paxinos, G. (2013). Paxinos and Franklin's The mouse brain in stereotaxic coordinates.

Gonzalez, D., Satriotomo, I., Miki, T., Lee, K.-Y., Yokoyama, T., Touge, T., ... Takeuchi, Y. (2006). Changes of parvalbumin immunoreactive neurons and GFAP immunoreactive astrocytes in the rat lateral geniculate nucleus following monocular enucleation. Neuroscience Letters, 395(2), 149-154. https://doi.org/https://doi.org/10.1016/j.neulet.2005.10.067

Halassa, M. M., Fellin, T., Takano, H., Dong, J.-H., \& Haydon, P. G. (2007). Synaptic Islands Defined by the Territory of a Single Astrocyte. The Journal of Neuroscience, 27(24), 6473 LP - 6477. https://doi.org/10.1523/JNEUROSCI.1419-07.2007

Hasel, P., Dando, O., Jiwaji, Z., Baxter, P., Todd, A. C., Heron, S., ... Hardingham, G. 
791

792

793

794

795

796

797

798

799

800

801

802

803

804

805

806

807

808

809

810

811

812

813

814

815

816

817

818

E. (2017). Neurons and neuronal activity control gene expression in astrocytes to regulate their development and metabolism. Nature Communications, 8, 15132. Retrieved from https://doi.org/10.1038/ncomms 15132

Hayakawa, K., Nakano, T., Irie, K., Higuchi, S., Fujioka, M., Orito, K., ... Fujiwara, M. (2010). Inhibition of reactive astrocytes with fluorocitrate retards neurovascular remodeling and recovery after focal cerebral ischemia in mice. Journal of Cerebral Blood Flow and Metabolism. https://doi.org/10.1038/jcbfm.2009.257

Hensch, T. K. (2005). Critical period plasticity in local cortical circuits. Nature Reviews Neuroscience, 6(11), 877-888. https://doi.org/10.1038/nrn1787

Hübener, M., \& Bonhoeffer, T. (2014). Neuronal Plasticity: Beyond the Critical Period. Cell, 159(4), 727-737. https://doi.org/https://doi.org/10.1016/j.cell.2014.10.035

Kim, S. Y., Hsu, J. E., Husbands, L. C., Kleim, J. A., \& Jones, T. A. (2018). Coordinated Plasticity of Synapses and Astrocytes Underlies Practice-Driven Functional Vicariation in Peri-Infarct Motor Cortex. The Journal of Neuroscience, 38(1), 93 LP - 107. https://doi.org/10.1523/JNEUROSCI.1295-17.2017

Kuter, K., Olech, Ł., \& Głowacka, U. (2018). Prolonged Dysfunction of Astrocytes and Activation of Microglia Accelerate Degeneration of Dopaminergic Neurons in the Rat Substantia Nigra and Block Compensation of Early Motor Dysfunction Induced by 6-OHDA. Molecular Neurobiology, 55(4), 3049-3066. https://doi.org/10.1007/s12035-017-0529-z

Lanjakornsiripan, D., Pior, B.-J., Kawaguchi, D., Furutachi, S., Tahara, T., Katsuyama, Y., ... Gotoh, Y. (2018). Layer-specific morphological and molecular differences in neocortical astrocytes and their dependence on neuronal layers. Nature Communications, 9(1), 1623. https://doi.org/10.1038/s41467-018-03940-3

Liauw, J., Hoang, S., Choi, M., Eroglu, C., Choi, M., Sun, G., ... Steinberg, G. K. (2008). Thrombospondins 1 and 2 are Necessary for Synaptic Plasticity and Functional Recovery after Stroke. Journal of Cerebral Blood Flow \& Metabolism, 28(10), 1722-1732. https://doi.org/10.1038/jcbfm.2008.65 
819 Lombaert, N., Hennes, M., Gilissen, S., Schevenels, G., Aerts, L., Vanlaer, R., ...

820 Arckens, L. (2018). 5-HTR 2A and 5-HTR 3A but not 5-HTR 1A antagonism impairs

821

822

823

824

825

826

827

828

829

830

831

832

833

834

835

836

837

838

839

840

841

842

843

844

845

846 the cross-modal reactivation of deprived visual cortex in adulthood. Molecular Brain. https://doi.org/10.1186/s13041-018-0404-5

Mariotti, L., Losi, G., Sessolo, M., Marcon, I., \& Carmignoto, G. (2016). The inhibitory neurotransmitter GABA evokes long-lasting $\mathrm{Ca} 2+$ oscillations in cortical astrocytes. Glia, 64(3), 363-373. https://doi.org/10.1002/glia.22933

Mataga, N., Fujishima, S., Condie, B. G., \& Hensch, T. K. (2001). ExperienceDependent Plasticity of Mouse Visual Cortex in the Absence of the Neuronal Activity-Dependent Marker\&lt;em\&gt;egr1/zif268\&lt;/em\&gt; The Journal of Neuroscience, 21(24), 9724 LP - 9732. https://doi.org/10.1523/JNEUROSCI.21-2409724.2001

Müller, C. M., \& Best, J. (1989). Ocular dominance plasticity in adult cat visual cortex after transplantation of cultured astrocytes. Nature, 342(6248), 427-430. https://doi.org/10.1038/342427a0

Nagai, J., Rajbhandari, A. K., Gangwani, M. R., Hachisuka, A., Coppola, G., Masmanidis, S. C., ... Khakh, B. S. (2019). Hyperactivity with Disrupted Attention by Activation of an Astrocyte Synaptogenic Cue. Cell, 177(5), 1280-1292.e20. https://doi.org/https://doi.org/10.1016/j.cell.2019.03.019

Navarrete, M., Perea, G., de Sevilla, D. F., Gómez-Gonzalo, M., Núñez, A., Martín, E. D., \& Araque, A. (2012). Astrocytes Mediate In Vivo Cholinergic-Induced Synaptic Plasticity. PLOS Biology, 10(2), e1001259. Retrieved from https://doi.org/10.1371/journal.pbio.1001259

Nikolakopoulou, A. M., Koeppen, J., Garcia, M., Leish, J., Obenaus, A., \& Ethell, I. M. (2016). Astrocytic Ephrin-B1 Regulates Synapse Remodeling Following Traumatic Brain Injury. ASN Neuro, 8(1), 1759091416630220. https://doi.org/10.1177/1759091416630220

Nys, J., Aerts, J., Ytebrouck, E., Vreysen, S., Laeremans, A., \& Arckens, L. (2014). The 
cross-modal aspect of mouse visual cortex plasticity induced by monocular enucleation is age dependent. Journal of Comparative Neurology. https://doi.org/10.1002/cne.23455

Nys, J., Smolders, K., Laramée, M.-E., Hofman, I., Hu, T.-T., \& Arckens, L. (2015). Regional Specificity of GABAergic Regulation of Cross-Modal Plasticity in Mouse Visual Cortex after Unilateral Enucleation. The Journal of Neuroscience, 35(32), 11174 LP - 11189. https://doi.org/10.1523/JNEUROSCI.3808-14.2015

Oberheim, N. A., Takano, T., Han, X., He, W., Lin, J. H. C., Wang, F., ... Nedergaard, M. (2009). Uniquely Hominid Features of Adult Human Astrocytes. The Journal of Neuroscience, 29(10), 3276 LP - 3287. https://doi.org/10.1523/JNEUROSCI.470708.2009

Parpura, V., \& Zorec, R. (2010). Gliotransmission: Exocytotic release from astrocytes. Brain Research Reviews, 63(1-2), 83-92. https://doi.org/10.1016/j.brainresrev.2009.11.008

Pascual-Leone, A., Amedi, A., Fregni, F., \& Merabet, L. B. (2005). THE PLASTIC HUMAN BRAIN CORTEX. Annual Review of Neuroscience, 28(1), 377-401. https://doi.org/10.1146/annurev.neuro.27.070203.144216

Paulsen, R. E., Contestabile, A., Villani, L., \& Fonnum, F. (1987). An In Vivo Model for Studying Function of Brain Tissue Temporarily Devoid of Glial Cell Metabolism: The Use of Fluorocitrate. Journal of Neurochemistry. https://doi.org/10.1111/j.14714159.1987.tb05674.x

Ronald Zielke, H., Zielke, C. L., Baab, P. J., \& Tyson Tildon, J. (2007). Effect of fluorocitrate on cerebral oxidation of lactate and glucose in freely moving rats. Journal of Neurochemistry, 101(1), 9-16. https://doi.org/10.1111/j.14714159.2006.04335.x

Saur, L., Baptista, P. P. A., De Senna, P. N., Paim, M. F., Nascimento, P. Do, Ilha, J., ... Xavier, L. L. (2014). Physical exercise increases GFAP expression and induces morphological changes in hippocampal astrocytes. Brain Structure and Function. 
https://doi.org/10.1007/s00429-012-0500-8

Scheyltjens, I., Vreysen, S., Van den Haute, C., Sabanov, V., Balschun, D., Baekelandt, V., \& Arckens, L. (2018). Transient and localized optogenetic activation of somatostatin-interneurons in mouse visual cortex abolishes long-term cortical plasticity due to vision loss. Brain Structure \& Function, 223(5), 2073-2095. https://doi.org/10.1007/s00429-018-1611-7

Scofield, M. D., Boger, H. A., Smith, R. J., Li, H., Haydon, P. G., \& Kalivas, P. W. (2015). Gq-DREADD Selectively Initiates Glial Glutamate Release and Inhibits Cue-induced Cocaine Seeking. Biological Psychiatry, 78(7), 441-451. https://doi.org/10.1016/j.biopsych.2015.02.016

Singh, S. K., Stogsdill, J. A., Pulimood, N. S., Dingsdale, H., Kim, Y. H., Pilaz, L.-J., ... Eroglu, C. (2016). Astrocytes Assemble Thalamocortical Synapses by Bridging NRX1\&\#x3b1; and NL1 via Hevin. Cell, 164(1), 183-196. https://doi.org/10.1016/j.cell.2015.11.034

Singh, S. K., Stogsdill, J. A., Pulimood, N. S., Dingsdale, H., Kim, Y. H., Pilaz, L.-J., ... Eroglu, C. (2016). Astrocytes Assemble Thalamocortical Synapses by Bridging NRX1a and NL1 via Hevin. Cell, 164(1-2), 183-196. https://doi.org/10.1016/j.cell.2015.11.034

Slezak, M., Kandler, S., Van Veldhoven, P. P., Bonin, V., \& Holt, M. G. (2018). Astrocytes integrate local sensory and brain-wide neuromodulatory signals. BioRxiv, 381434. https://doi.org/10.1101/381434

Sonoda, K., Matsui, T., Bito, H., \& Ohki, K. (2018). Astrocytes in the mouse visual cortex reliably respond to visual stimulation. Biochemical and Biophysical Research Communications, 505(4), 1216-1222. https://doi.org/https://doi.org/10.1016/j.bbrc.2018.10.027

Stogsdill, J. A., Ramirez, J., Liu, D., Kim, Y. H., Baldwin, K. T., Enustun, E., ... Eroglu, C. (2017). Astrocytic neuroligins control astrocyte morphogenesis and synaptogenesis. Nature, 551, 192. Retrieved from 
https://doi.org/10.1038/nature24638

Teng-nan, L., Gyoeng-Moon, K., Jean-Ju, C., Wai-Mui, C., Y., H. Y., \& Y., H. C. (2003). Differential Regulation of Thrombospondin-1 and Thrombospondin-2 After Focal Cerebral Ischemia/Reperfusion. Stroke, 34(1), 177-186. https://doi.org/10.1161/01.STR.0000047100.84604.BA

van Brussel, L., Gerits, A., \& Arckens, L. (2009). Identification and localization of functional subdivisions in the visual cortex of the adult mouse. Journal of Comparative Neurology, 514(1), 107-116. https://doi.org/10.1002/cne.21994

Van Brussel, L., Gerits, A., \& Arckens, L. (2011). Evidence for cross-modal plasticity in adult mouse visual cortex following monocular enucleation. Cerebral Cortex. https://doi.org/10.1093/cercor/bhq286

Van der Perren, A., Toelen, J., Carlon, M., Van den Haute, C., Coun, F., Heeman, B., ... Baekelandt, V. (2011). Efficient and stable transduction of dopaminergic neurons in rat substantia nigra by rAAV 2/1, 2/2, 2/5, 2/6.2, 2/7, 2/8 and 2/9. Gene Therapy, 18(5), 517-527. https://doi.org/10.1038/gt.2010.179

Vreysen, S., Scheyltjens, I., Laramée, M.-E., \& Arckens, L. (2017). A Tool for BrainWide Quantitative Analysis of Molecular Data upon Projection into a Planar View of Choice. Frontiers in Neuroanatomy. https://doi.org/10.3389/fnana.2017.00001

Weber, B., \& Barros, L. F. (2015). The Astrocyte: Powerhouse and Recycling Center. Cold Spring Harbor Perspectives in Biology, 7(12), a020396. https://doi.org/10.1101/cshperspect.a020396

Xin, W., \& Bonci, A. (2018). Functional Astrocyte Heterogeneity and Implications for Their Role in Shaping Neurotransmission. Frontiers in Cellular Neuroscience, 12, 141. https://doi.org/10.3389/fncel.2018.00141

Yashiro, K., Corlew, R., \& Philpot, B. D. (2005). Visual Deprivation Modifies Both Presynaptic Glutamate Release and the Composition of Perisynaptic/Extrasynaptic NMDA Receptors in Adult Visual Cortex. The Journal of Neuroscience, 25(50), 11684 LP - 11692. https://doi.org/10.1523/JNEUROSCI.4362-05.2005 
Zhang, Y., \& Barres, B. A. (2010). Astrocyte heterogeneity: an underappreciated topic in neurobiology. Current Opinion in Neurobiology, 20(5), 588-594. https://doi.org/https://doi.org/10.1016/j.conb.2010.06.005

Zielke, H. R., Zielke, C. L., \& Baab, P. J. (2009). Direct measurement of oxidative metabolism in the living brain by microdialysis: A review. Journal of Neurochemistry. https://doi.org/10.1111/j.1471-4159.2009.05941.x

\section{Figure legends}

\section{Figure 1. Illustration of experimental setup.}

a. Overview illustrating the timeline of stereotactic injections and manipulations of all monocular enucleation (ME)-related experiments. All mice had normal vision until the age of P120 when ME was performed and the animals were left to recover for 7 weeks (7wME). For the fluorocitrate experiment, mice received a stereotactic injection of fluorocitrate or saline in V1 at P120 (arrowhead timeline), prior to ME. For the chemogenetics experiment, mice were stereotactically injected with the viral vector constructs in V1 at P105 (arrowhead timeline). Animals in the mCherry, hM3Dq-mCherry and hM4Di-mCherry group received daily injections of $5 \mathrm{mg} / \mathrm{kg} \mathrm{CNO}$ for 7 weeks. b. Illustration of the interareal borders delineating the different visual cortex regions based on Nissl staining, and overlaid on the matching zif268 in situ hybridization pattern for sections of interest of each individual mouse. Delineated visual cortex regions from lateral to medial: V2L, V1, and V2M with the distinction between monocular $(\mathrm{m})$ and binocular (b) segments. The binocular zone $(\mathrm{Bz})$ consists of the V2Lb and the $\mathrm{V} 1 \mathrm{~b}$ area while the monocular zone $(\mathrm{Mz})$ contains the V1m and the V2M area. Cortical layers are indicated with Roman numbers: I-VI. c. Strategy of segmentation of cortical zif268-mRNA labeling for OD calculation of the different visual cortex regions in the supra- and infragranular layers to create line graph representations and allow statistical analysis concerning regional changes in neuronal activity. Illustration of the transformation of OD values to a grayscale heat map used for top view representation of the spatial zif268 expression pattern indicative of the neuronal reactivation. d. Supra- and infragranular area masks of 
960 the primary (V1) and extrastriate visual regions (POR: Postrhinal area; LI: 961 Laterointermediate area; LM: Lateromedial area; LLA: Laterolateral anterior area; AL: 962 Anterolateral area; RL: Rostrolateral area; A: Anterior area; AM: Anteromedial area; PM: 963 Posteromedial area) delineating the monocular (white) and the binocular (gray) zones. 964 Red arrows indicate the direction of binocular zone expansion and monocular zone 965 reactivation during the plasticity recovery phases following enucleation. Axis units in mm 966 from midline (x-axis) and bregma (y-axis).

Figure 2. Time-dependent effect of monocular enucleation on the density and layer 969 distribution pattern of Aldh1l1-positive astrocytes in the monocular and the binocular zone of the deprived visual cortex.

a. Representative images showing Aldh1/1-positive astrocytes across all layers of the visual cortex in control normal sighted (left) and 3-day enucleated mice (3dME) (right). Scale bar: $50 \mu \mathrm{m}$. b. High magnification picture showing clear protoplasmic astrocyte labeling based on typical star-shaped cellular morphology in control normal sighted (top) and 3dME mice (bottom). Scale bar: $10 \mu \mathrm{m}$.

c. Astrocyte density in the monocular zone at different times post-enucleation represented as astrocytes $/ \mathrm{mm}^{2}$. One-way ANOVA, Bonferroni post hoc tests, ${ }^{* * *} p$-value $<0,001$. d. Astrocyte layer distribution pattern in the monocular zone for all analyzed conditions. Two-way ANOVA, Bonferroni post hoc tests. e. Astrocyte density in the binocular zone at different times post-enucleation represented as astrocytes $/ \mathrm{mm}^{2}$. One-way ANOVA, Bonferroni post hoc tests, * $p$-value<0,05; ${ }^{* *} p$ value $<0,01 ;{ }^{* * *} p$-value $<0,001$. f. Astrocyte layer distribution pattern in the binocular zone for all analyzed conditions. Two-way ANOVA, Bonferroni post hoc tests, * $p$-value<0,05; *** $p$-value $<0,001$. ( $n=6$ for all conditions). 3dME, 3 days post-enucleation; 3wME, 3 weeks post-enucleation; 7wME, 7 weeks post-enucleation.

Figure 3. Fluorocitrate injection leads to a localized, selective and reversible silencing of visual cortex astrocytes. a. Intracranial injection of fluorocitrate leads to a local drop in Aldh1l1-positive astrocytes at one day post-injection in V1. White rectangle: 
injection site, Blue: DAPI, Red: Aldh1l1. b. Fluorocitrate does not affect the surrounding microglia (TMEM119) or neurons (NeuN) at one day post-injection Blue: DAPI, Green: NeuN, Red: TMEM119. (a, b illustrate data from adjacent sections). c. Three days postinjection, the effect of fluorocitrate is largely reversed as Aldh1/1- and GFAP-positive astrocytes are again present at the injection site. Blue: DAPI, Green: GFAP, Red: Aldh1l1. Scale bar: $500 \mu \mathrm{m}$.

\section{Figure 4. Analysis of the long-term effect of a single fluorocitrate injection on} neuronal recovery after monocular enucleation. a. Top view representations of the spatial neuronal reactivation profile based on zif268 expression levels in 7-week enucleated $(7 \mathrm{WME})$, fluorocitrate treated or saline injected mice. Left: top view supragranular layers, Right: top view infragranular layers, *: injection site. b. Representative images of zif268-mRNA labeled coronal sections surrounding bregma level $-3.50 \mathrm{~mm}$ and corresponding pseudocolor representations. Calibration bar: 0 (high) -255 (low), Scale bar: $1 \mathrm{~mm}$. c. Line graph representations of the relative zif268 expression measured as the averaged OD value per segment from lateral to medial for saline (black) and fluorocitrate (blue) injected 7wME mice. Zif268 expression levels in the supragranular layers II/III and IV (left) and the infragranular layers V and VI (right) are displayed for the monocular (V1m,V2M) and binocular zone (V2Lb, V1b) (separated by dotted vertical lines, in register with black arrowheads in b). Error bars represent SEM. $\mathbf{d}$. Comparison of averaged zif268 OD values for the binocular, monocular and V1 region of the deprived visual cortex in fluorocitate (blue) or saline (black) injected 7wME mice, separately analyzed for the supra- and infragranular layers. Mann-Whitney $U$ test, * $p$ value $<0,05, n=6$ for all conditions.

\section{Figure 5. Astrocyte specific expression and functional validation of the DREADD} constructs. a. Coronal sections illustrating co-labeling between mCherry (GFABC1DAAV2.5 viral vector) with astrocyte specific cytoplasmic (Aldh1l1, GFAP) and plasma membrane markers (Connexin43, Kir4.1) in V1. Blue: DAPI, Red: mCherry reporter gene, Green: cellular marker. b. No co-labeling was observed between the mCherry and 
1019 neuronal (NeuN), interneuronal (Parvalbumin), microglial (TMEM119) or oligodendrocytic 1020 (Olig2) cellular markers. Blue: DAPI, Red: mCherry reporter gene, Green: cellular marker. c. Acute CNO administration leads to induction of Fos expression in hM4Di- and hM3Dq1022 expressing astrocytes but not in control (mCherry -expressing) astrocytes. White arrows: 1023 Fos expressing astrocytes, Blue: Fos, Red: mCherry reporter gene, Green: GFAP. Scale 1024 bar: $50 \mu \mathrm{m}$.

1025 Figure 6. Comparison of the neuronal reactivation patterns after long-term 1026 chemogenetic astrocyte activation following monocular enucleation.

1027 a. Top view representations of the spatial neuronal reactivation pattern based on zif268 1028 expression levels in hM3Dq-mCherry, mCherry and hM4Di-mCherry expressing mice after 7 weeks of daily CNO injections following ME. Left: top view supragranular layers, Right: top view infragranular layers, *: injection site. b. Representative images of zif268 mRNA coronal sections surrounding bregma $-3.50 \mathrm{~mm}$ with their corresponding pseudocolor representation and mCherry expression confirming V1 vector localization. Calibration bar: 0 (high) - 255 (low). Scale bar: $1 \mathrm{~mm}, 100 \mu \mathrm{m}$ (detail mCherry expression) c. Line graph representations of the relative zif268 expression measured as the averaged $\mathrm{OD}$ value per segment from lateral to medial for hM3Dq-mCherry (green), mCherry (black) and hM4Di-mCherry (red)-expressing mice. Zif268 expression levels of the supragranular layers II/III and IV (left) and the infragranular layers V and VI (right) are displayed for the monocular (V1m,V2M) and binocular zone (V2Lb, V1b) (separated by dotted vertical lines, in register with black arrowheads in b). Error bars represent SEM. $\mathbf{d}$. Comparison of the averaged zif268 OD values in the measured regions for hM3DqmCherry (green), mCherry (black) and hM4Di-mCherry (red)-expressing mice. Only bar 1042 graphs showing significant differences are presented. Mann-Whitney $U$ test, ${ }^{*} p$ 1043 value $<0,05,{ }^{* *} \mathrm{p}$-value $<0,01, \mathrm{n}=7$ for all conditions. 


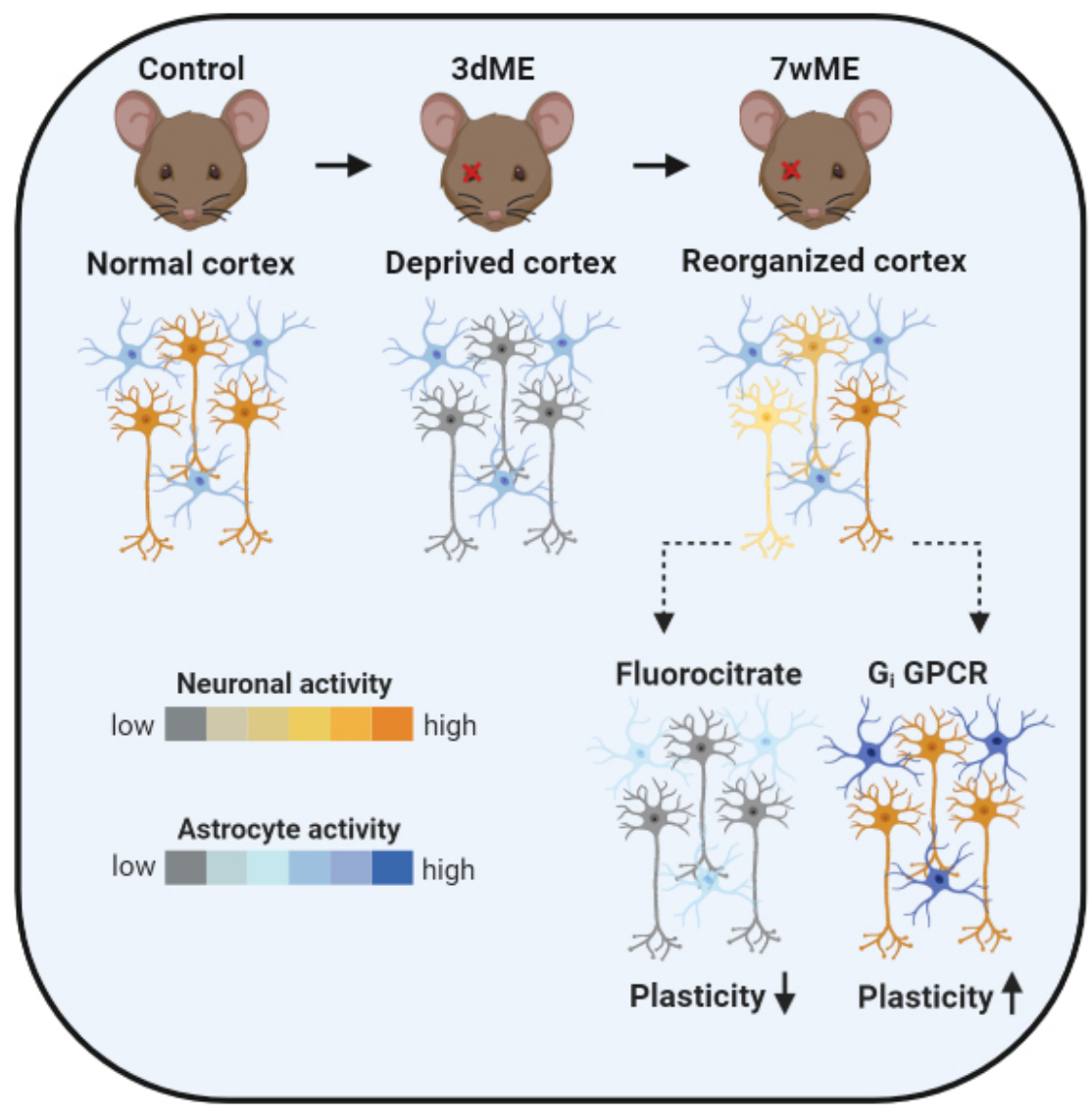

Table of contents image 


\begin{tabular}{|c|c|c|}
\hline Page 39 Briø3ary Antibody & GLIA Company & Dilution \\
\hline rabbit anti-Aldh1/1 & AB87117, Abcam (Cambridge, UK) & $1: 200$ \\
\hline rabbit anti-GFAP & PA5-16291, Thermofisher Scientific (Rockford, USA) & $1: 500$ \\
\hline mouse anti-GFAP & MA5-12023, Thermofisher Scientific (Rockford, USA) & $1: 500$ \\
\hline mouse anti-NeuN & MAB377, Milipore Corp (CA, USA) & $1: 200$ \\
\hline rabbit anti-TMEM119 & AB209064, Abcam (Cambridge, UK) & $1: 500$ \\
\hline rabbit anti-Connexin43 & 71-0700, Thermofisher Scientific (Rockford, USA) & $1: 500$ \\
\hline rabbit anti-Kir4.1 & APC035, Alomone Labs (Jerusalem, Israel) & $1: 500$ \\
\hline mouse anti-Parvalbumin & PV235, Swant (Marly, Switzerland) & $1: 10000$ \\
\hline rabbit anti-Olig2 & AB109186, Abcam (Cambridge, UK) & $1: 500$ \\
\hline mouse anti-RFP & 200-301-379, Tebu-Bio (Boechout, Belgium) & $1: 2000$ \\
\hline rabbit anti-RFP & 600-401-379, Tebu-Bio (Boehout, Belgium) $\square$ & $1: 2000$ \\
\hline RFP Booster & RBA594-100, ChromoTek (Planegg-Martinsried, Germany) & $1: 500$ \\
\hline rabbit anti-Fos & In house made & $1: 5000$ \\
\hline Secondary Antibody & Company & Dilution \\
\hline Goat anti-rabbit Alexa Fluor 488 & AB150085, Abcam (Cambridge, UK) & $1: 250$ \\
\hline Goat anti-rabbit Alexa Fluor 594 & AB150088, Abcam (Cambridge, UK) & $1: 250$ \\
\hline Goat anti-mouse Alexa Fluor 488 & AB150117, Absam (Cambridge UK) & $1: 250$ \\
\hline Goat anti-mouse Alexa Fluor 594 & AB150120, Abcam (Cambridge, UK) & $1: 250$ \\
\hline Goat anti-mouse Cy5 & AB6563, Abcam (Cambridge, UK) & $1: 250$ \\
\hline
\end{tabular}


a

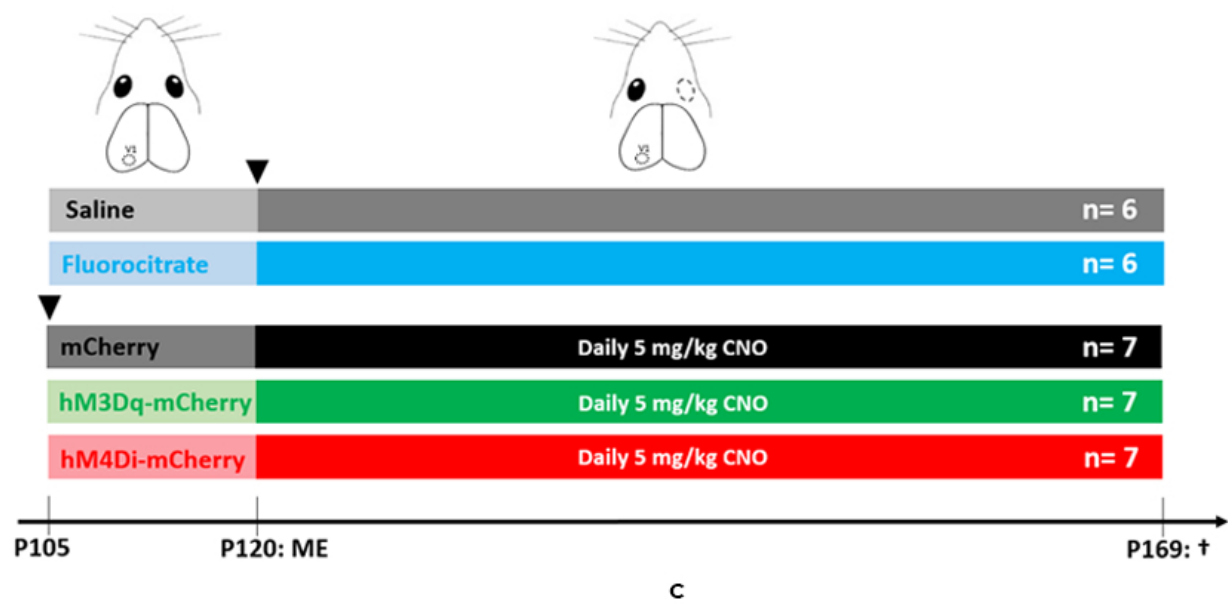

b
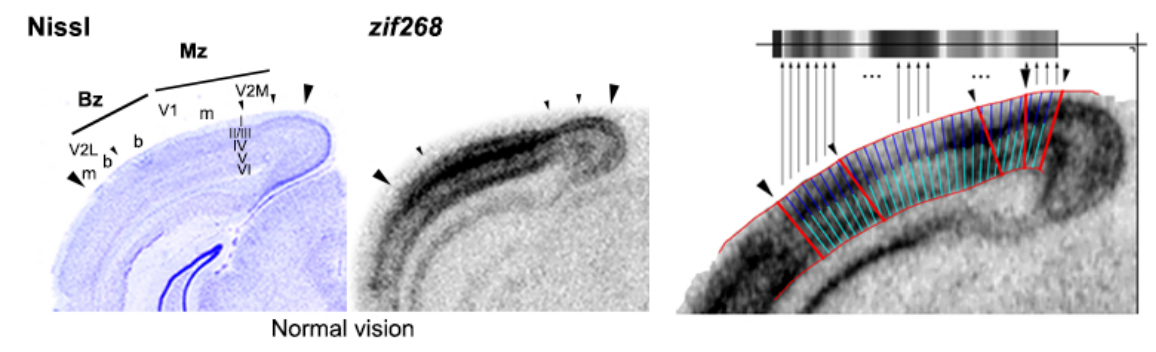

d

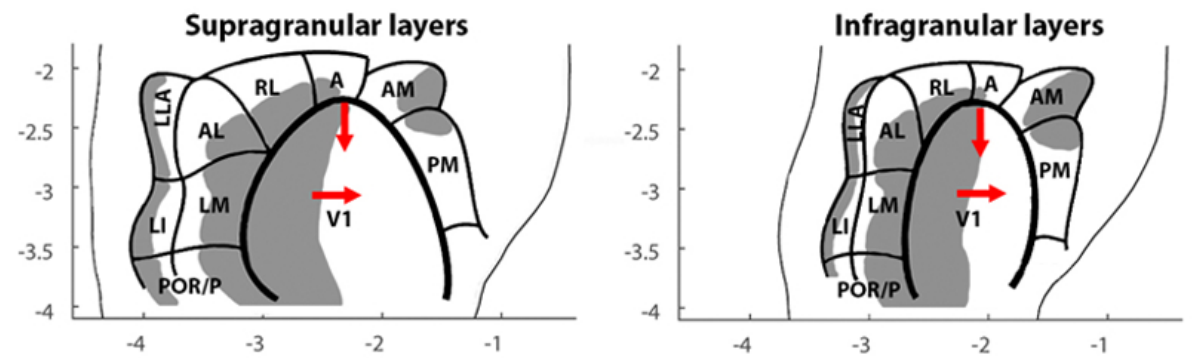

Figure 1. Illustration of experimental setup. 


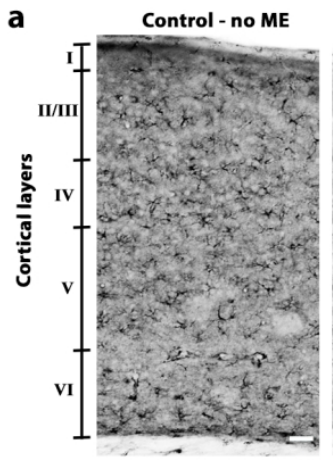

C

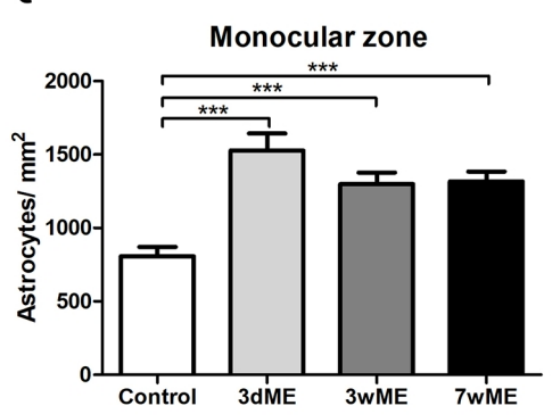

e

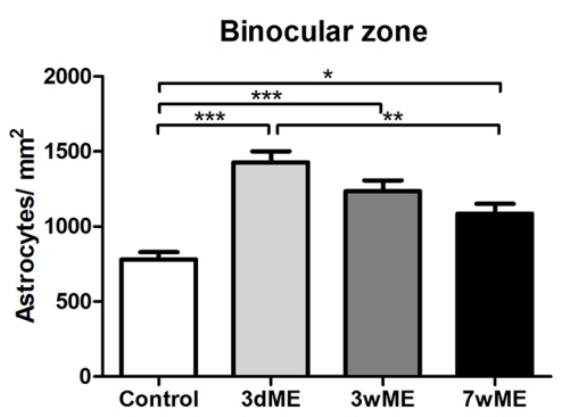

b

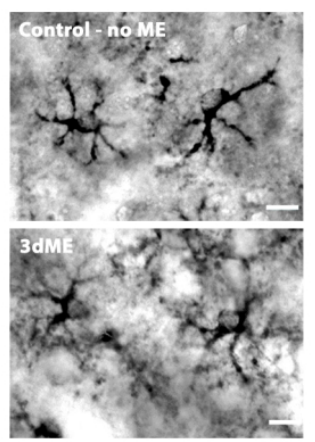

d

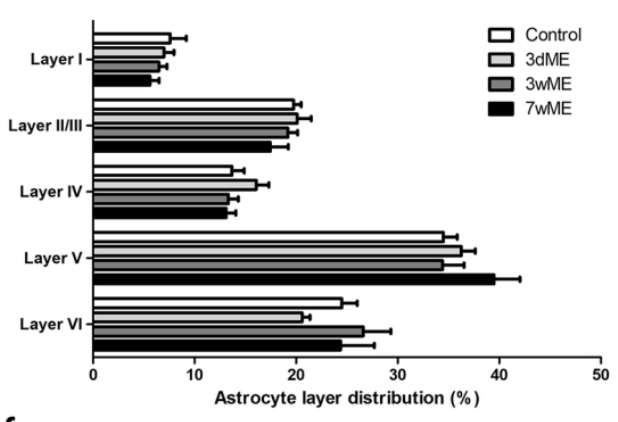

f

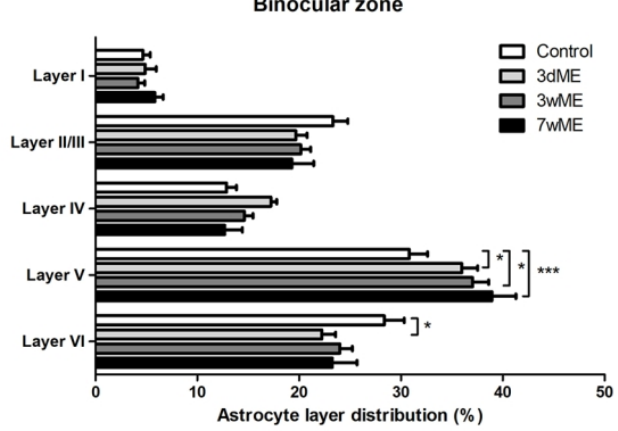

Figure 2. Time-dependent effect of monocular enucleation on the density and layer distribution pattern of Aldh111-positive astrocytes in the monocular and the binocular zone of the deprived visual cortex. 


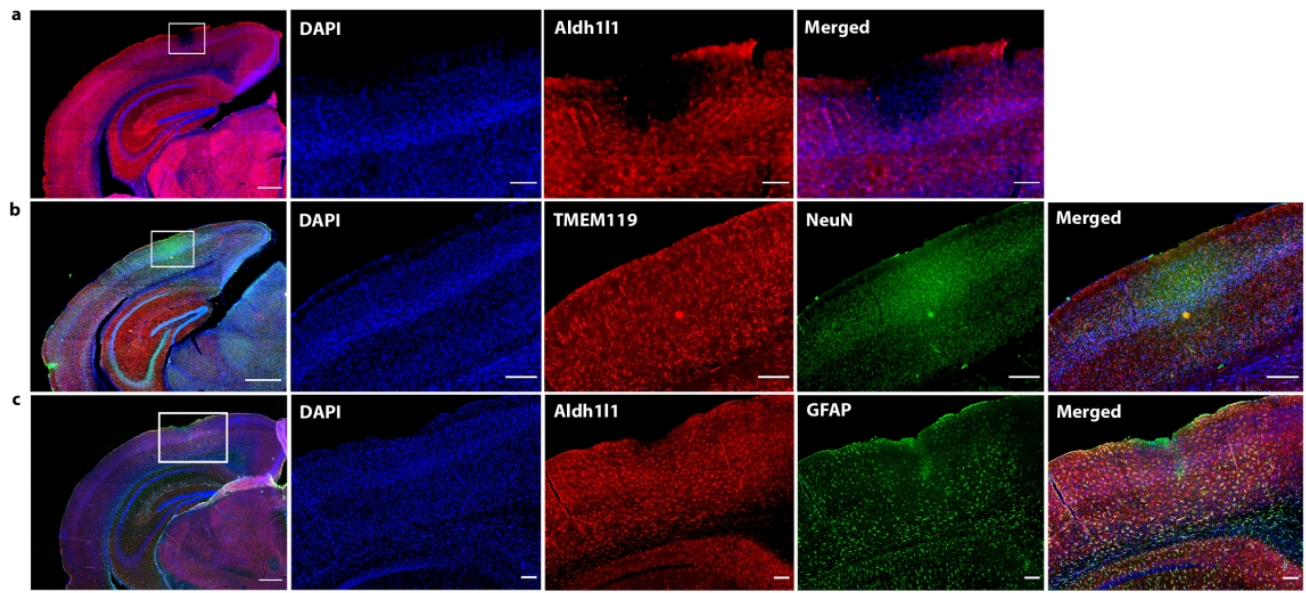

Figure 3. Fluorocitrate injection leads to a localized, selective and reversible silencing of visual cortex astrocytes. 


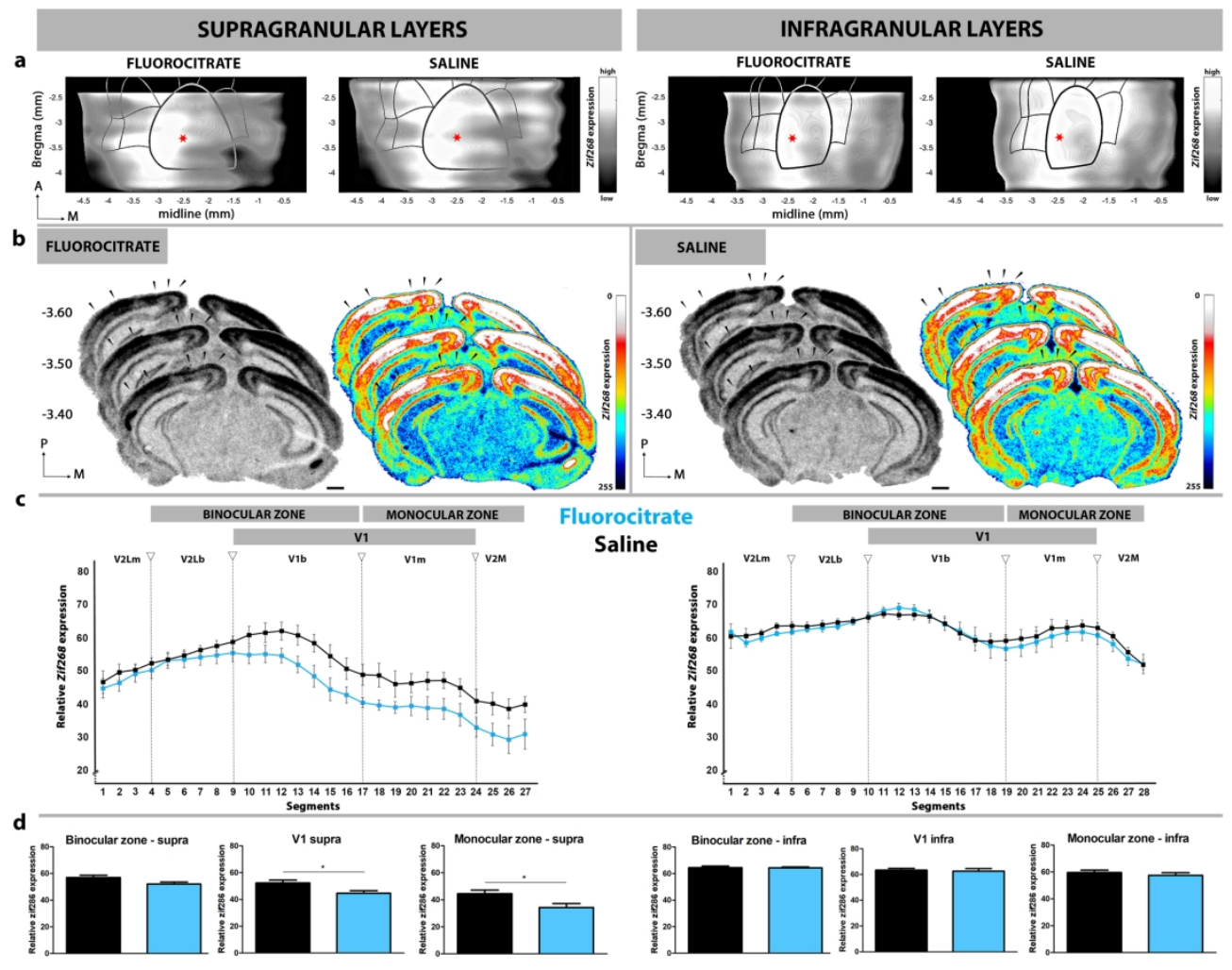

Figure 4. Analysis of the long-term effect of a single fluorocitrate injection on neuronal recovery after monocular enucleation. 


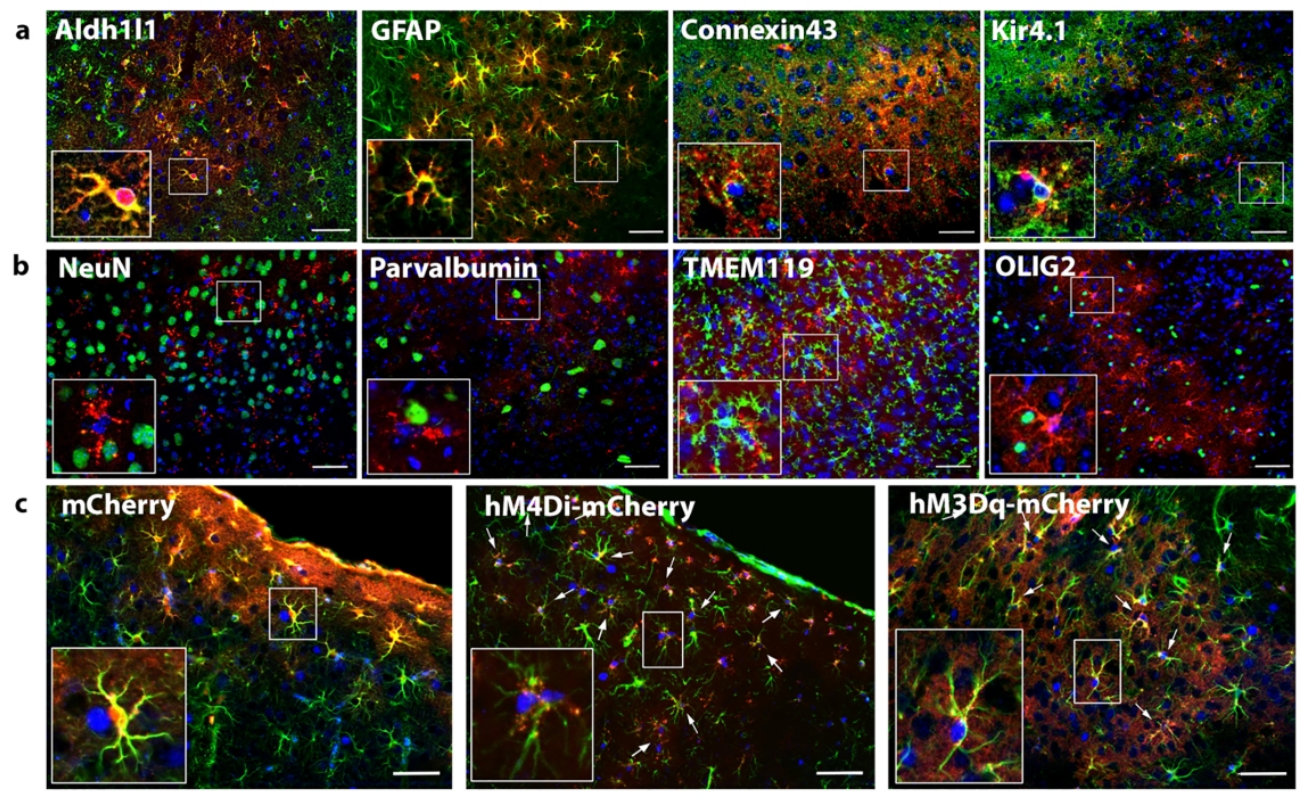

Figure 5. Astrocyte specific expression and functional validation of the DREADD constructs. 


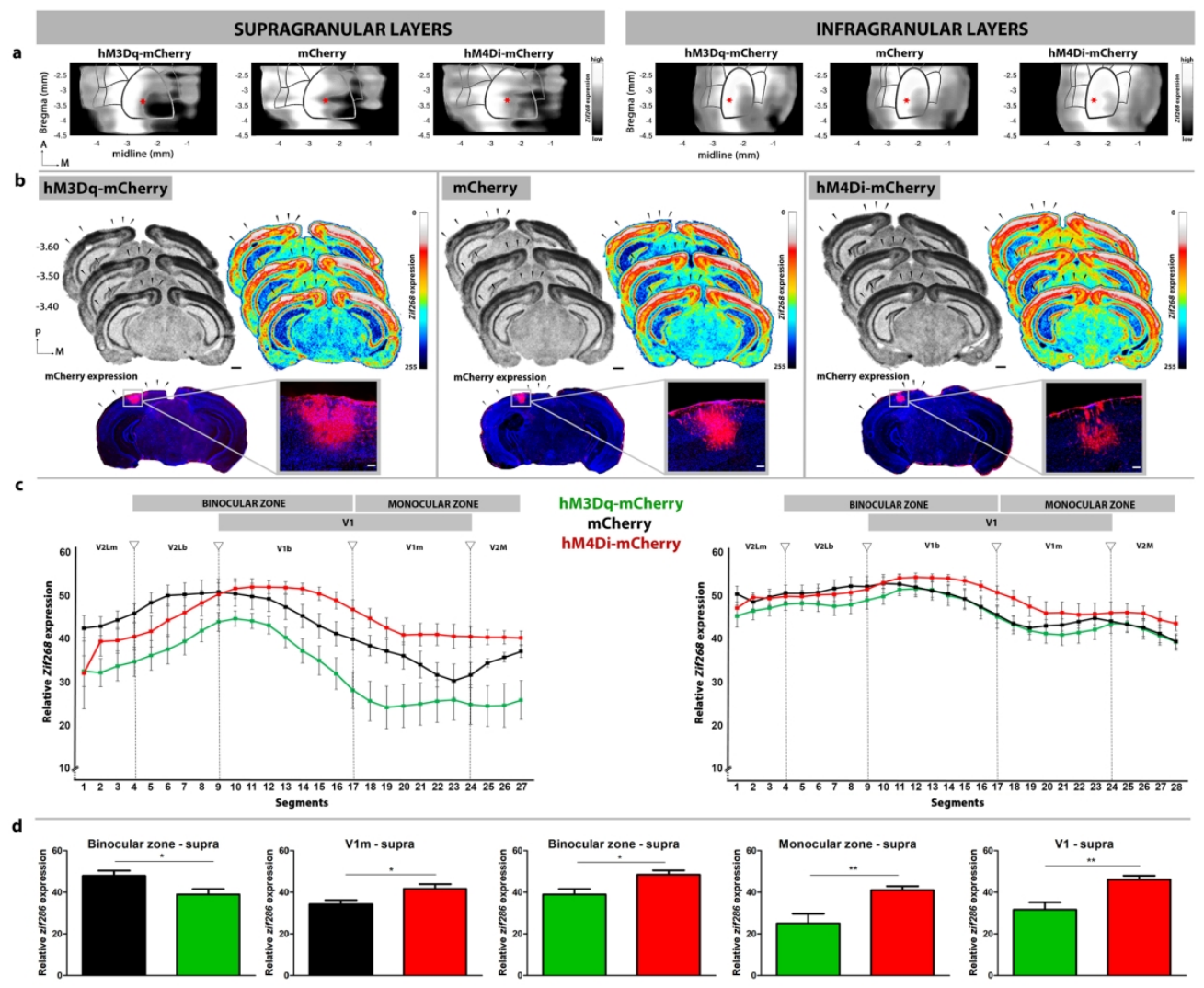

Figure 6. Comparison of the neuronal reactivation patterns after long-term chemogenetic astrocyte activation following monocular enucleation. 


\section{Supplementary figure legends}

Supplementary figure 1. Neuronal and microglial cells are not affected by fluorocitrate at one day post-injection. No differences in neuronal or microglial cell shape or distribution can be observed one day post-injection between saline- (a) or fluorocitrate- (b) injected mice. Red:

TMEM119, Blue: DAPI, Green: NeuN. Scale bar: $50 \mu \mathrm{m}, 10 \mu \mathrm{m}$.

Supplementary figure 2. Cortical saline injection does not suppress the surrounding Aldh1l1positive astrocyte population. a. At one day post-injection Aldh1/1-positive astrocytes remain detectable at the injection site. White rectangle: injection site, Blue: DAPI, Red: Aldh1l1. b. GFAPpositive astrocytes are detectable in the visual cortex three days post-injection of saline as a result of the intracranial injection. Blue: DAPI, Red: Aldh1l1, Green: GFAP. Scale bar: $500 \mu \mathrm{m}$.

Supplementary figure 3. Monocular enucleation is not inducing neuronal degeneration or reactive astrogliosis. a. Overview of the neuronal and reactive astrocyte population in visual cortex of control normal sighted P120 mouse. Red: NeuN, Green: GFAP. b. Three days post ME, the neuronal cell distribution remains intact and no reactive astrocytes are observed in the visual cortex. Red: NeuN, Green: GFAP. Scale bar: $500 \mu \mathrm{m}$. 


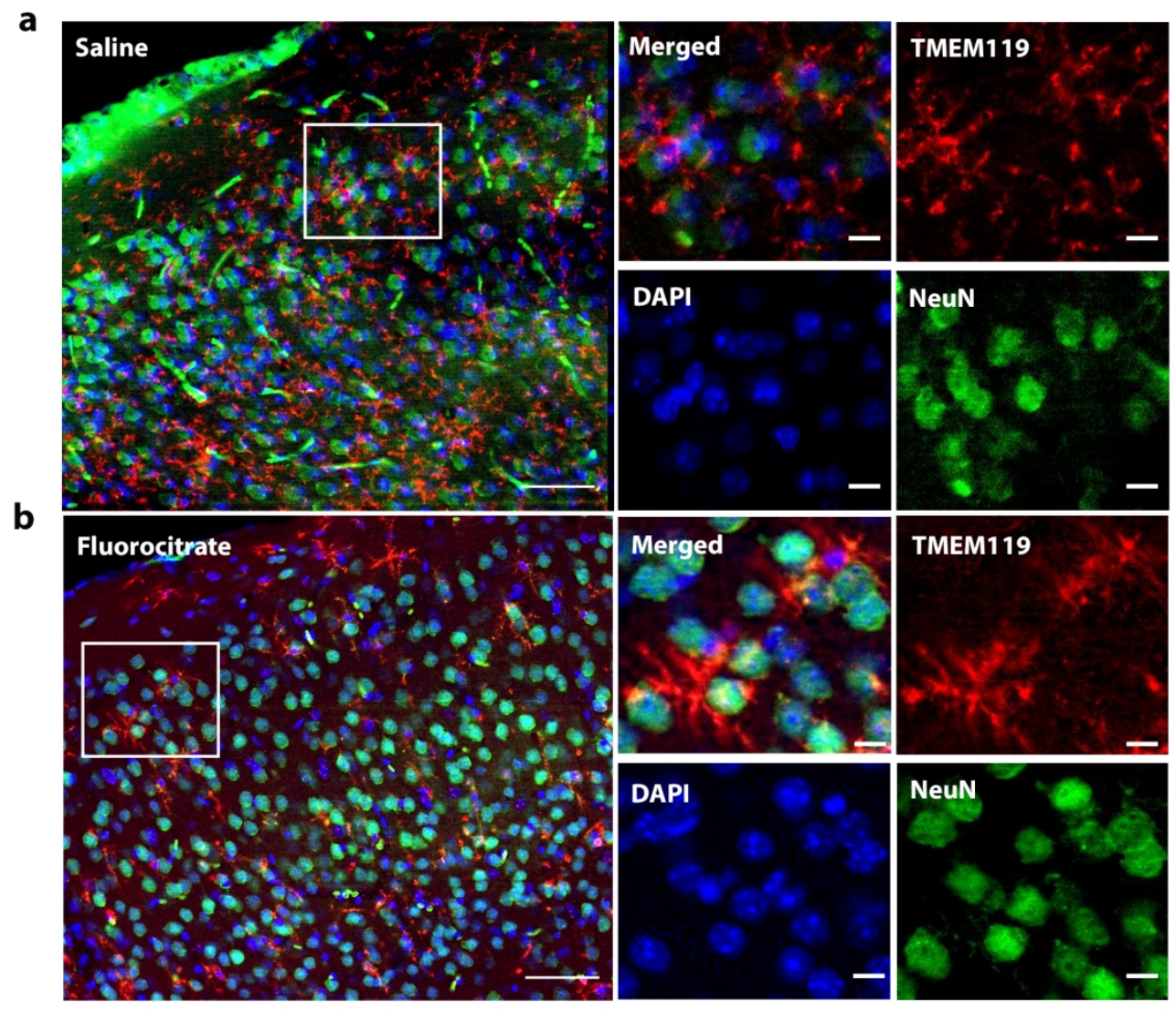




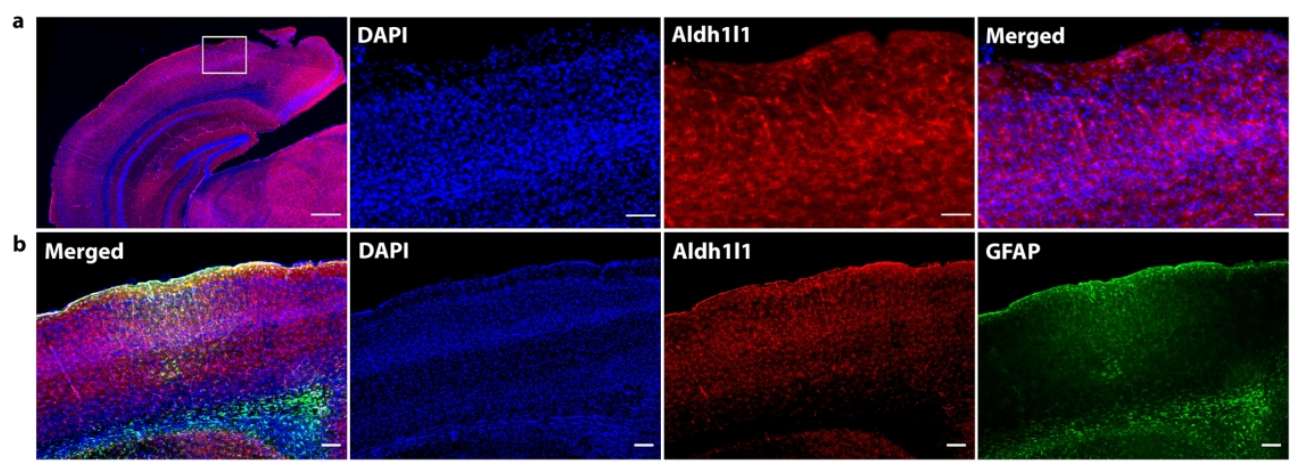

John Wiley \& Sons, Inc. 


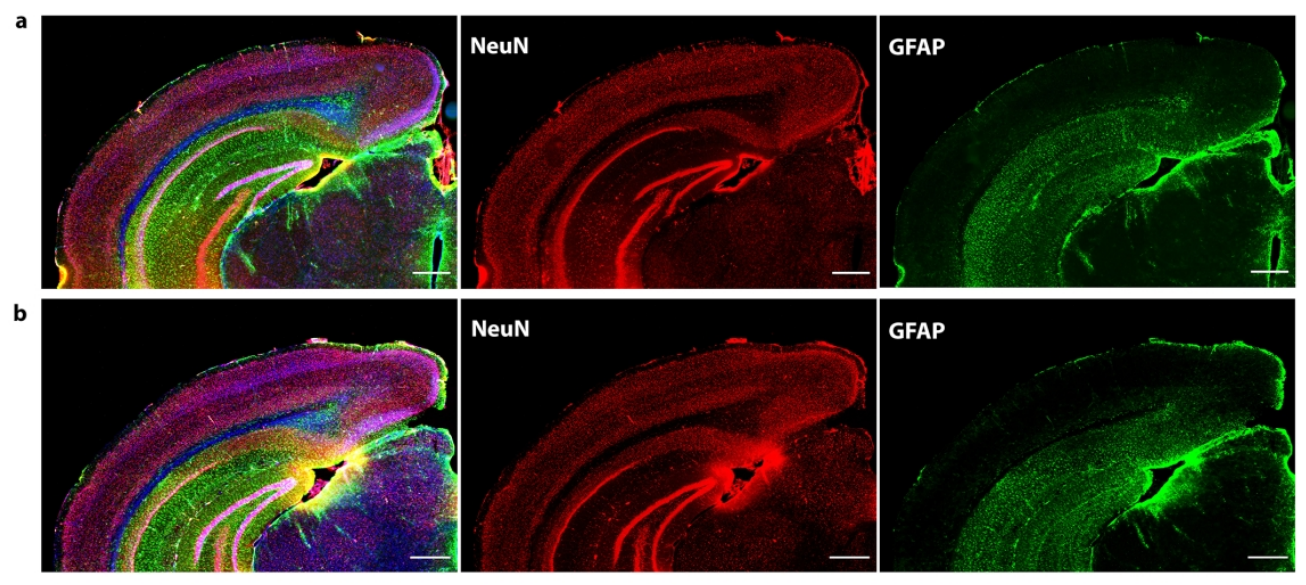

John Wiley \& Sons, Inc. 


\section{Leuven, February, 2020}

\section{Cover letter Revision}

Dear editor,

Thank you for the opportunity to revise our manuscript: "Astrocytes shape the plastic response of adult cortical neurons to vision loss" - ID GLIA-00394-2019. We appreciate the thorough review and constructive suggestions that substantially improved our manuscript.

To meet the editors requests, we have included Main points to our manuscript as well as a summary graphic for the online table of contents. The manuscript was carefully proofred and figures were adapted to match size requirements.

Following this letter you can find the reviewers comments marked in blue and our responses marked in black with reference to how and where the manuscript was edited. In our opinion, we have provided the reviewers with answers and additional figures to meet their requests.

We want to thank you and the Editorial board for your time and consideration.

Kind regards,

Lutgarde Arckens

Head Laboratory of Neuroplasticity and Neuroproteomics

Division of Animal Physiology and Neurobiology, Department of Biology

KU Leuven

Belgium 
12-Dec-2019

Dear Miss Hennes:

Manuscript ID GLIA-00394-2019 entitled "Astrocytes shape the plastic response of adult cortical neurons to vision loss" has been reviewed. The comments of the reviewer(s) are included at the end of this letter.

There is interest in your manuscript, but as you can see from the reviews (below), substantial revisions are recommended and the paper must undergo further peer review. Therefore, I invite you to revise your manuscript, taking into account the suggestions made by the reviewers.

Before you resubmit your paper, please carefully proof-read the manuscript to minimize typographical, grammatical, and bibliographical errors. In addition, check to make sure that all abbreviations are defined.

In addition, please read the author guidelines carefully: https://onlinelibrary.wiley.com/page/journal/10981136/homepage/forauthors.html

We realized that you did not provide us with main points and a $\mathrm{TOCl}$ what most authors do:

The table of contents entry for each article published in GLIA comprises of main points and an accompanying graphic:

Main Points are the most important findings and/or conclusions of the article (or review), stated concisely. Main Points will be displayed online immediately below the article's title (they will not appear in the printed version of the paper). Main Points should consist of 2 to 3 short sentences and should not exceed 250 characters (including spaces). These are required for all papers published in GLIA, and must be included in the title page.

Table of Contents Image (TOCI) is a summary graphic that appears in the online table of contents and in Early View articles. It is intended to illustrate and complement the Main Points. We strongly recommend submission of a $\mathrm{TOCl}$ but it is not mandatory. Authors should ideally upload the $\mathrm{TOCl}$ at the point of submission.

TOCI requirements:

Resolution: 300 d.p.i. for color/half tone and 800 d.p.i. for line work

File format: .tiff/.tif or .eps

Dimensions: 2.25 in / $5.7 \mathrm{~cm} / 215$ pixels (width) by $2 \mathrm{in} \mathrm{/} 5 \mathrm{~cm} / 192$ pixels (height)

Additionally please add a word count and running title to your cover page.

To submit your revised manuscript, log into https://mc.manuscriptcentral.com/glia and enter your Author Center, where you will find your manuscript title listed under 
"Manuscripts with Decisions." Under "Actions," click on "Create a Revision." Your manuscript number has been appended to denote a revision.

When submitting your revised manuscript, please respond to the comments made by the reviewer(s) in the space provided. You can use this space to document any changes you make to the original manuscript. In order to expedite the processing of the revised manuscript, please be as specific as possible in your responses to the reviewer(s).

Please realize that this invitation to resubmit is not a guarantee of acceptance.

IMPORTANT: Please submit files (Text, Tables, Figures) separately, conforming to the following formats: DOC, RTF (Text/Tables) EPS, TIF (Tables/Figures). On the Title Page please include the exact number of words(by section, including legends and bibliography), figures and tables in the article, plus the total word count.

Once again, thank you for submitting your manuscript to GLIA and I look forward to receiving your revision.

Sincerely,

Prof. Helmut Kettenmann

Editor-in-Chief, GLIA

\section{Point-by-point reply to the comments of the reviewers:}

\section{Reviewer: 1}

Comments to the Author

This manuscript by Hennes et al. raises the interesting research question, if and how astrocytes mediate processes of neuronal plasticity in the deprived visual cortex after monocular enucleation (ME). The authors observed ME-induced upregulation in the density of Aldh1/1-positive astrocytes up to 7 weeks after the injury. To test, whether this increased density of astrocytes affects the functional reorganization processes, they intracranially applied fluororocitrate at the time of ME to metabolically silence this type of glial cells in the visual cortex. By analyzing the expression pattern of the neuronal activity marker Zif-286 through in situ hybridization experiments they showed that metabolic activity of astrocytes is required to observe the previously observed neuronal hyperactivity as measured by an increased Zif-286 expression. Using a chemogenetic approach the authors further showed that a chronic stimulation of the Gi- but not of Gq GPCR in astrocytes can boost cortical reorganization. The authors conclude that astrocytes play an important role for functional reorganization of the visual cortex after ME via Gisignaling, and they suggest this mechanism as an interesting therapeutic pathway for brain plasticity and repair.

The manuscript is carefully written, methodically clean and the presented experiments are convincing. Since a peripheral, unilateral injury forces the visual system to perform its sensory tasks in an altered mode of using one-instead of two-eyes, the present ME model 
is ideal to study the role of astrocytes for processes of functional recruitment and reorganization in the visual cortex. The results are new with respect to the visual cortex and scientifically very interesting.

\section{Minor comments:}

1. One minor comment refers to Fig. 3. I had some difficulties to understand the details of the immunohistochemical pictures in Fig. 3a, b and c. Normally, the antibody overlap "merged" photograph is shown on the right of each row as the summary picture of the single antibody data shown in the other pictures on the left of the same row (like in Fig. 3a.). However, in Fig. 3b,c it is presented here rather mixed and confusing. I suggest to redo the figure and always present the "merged" data on the right of each row.

We apologize for the confusion and adapted the figure in line with the reviewer's comment. For completeness we also added the new figure 3 and legend below.
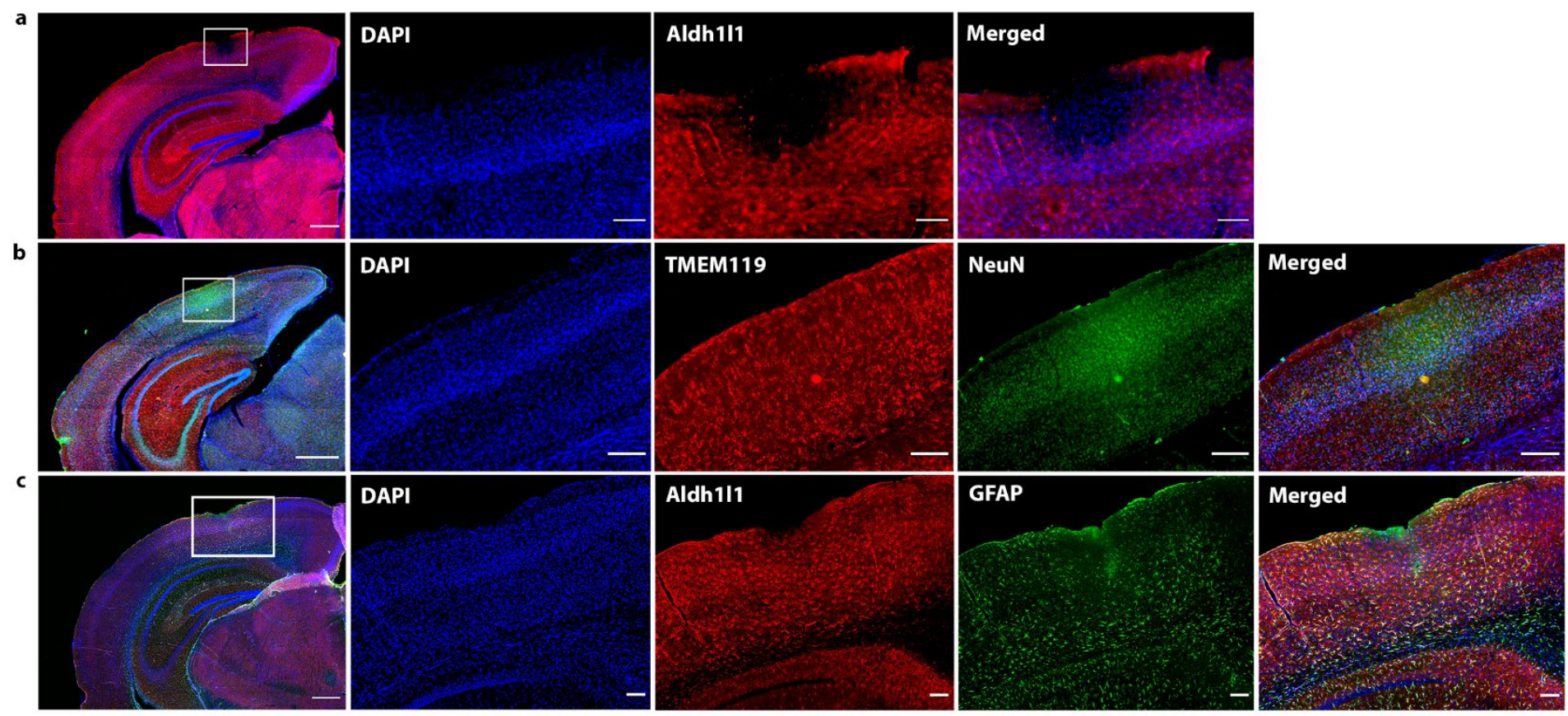

Figure 3. Fluorocitrate injection leads to a localized, selective and reversible silencing of visual cortex astrocytes. a. Intracranial injection of fluorocitrate leads to a local drop in Aldh1/1-positive astrocytes at one day post-injection in V1. White rectangle: injection site, Blue: DAPI, Red: Aldh1l1. b. Fluorocitrate is not affecting the surrounding microglia (TMEM119) or neurons (NeuN) or microglia at one day post-injection Blue: DAPI, Green: NeuN, Red: TMEM119. (a, b illustrate data from adjacent sections). Blue: DAPI, Green: NeuN, Red: TMEM119. c. Three days post-injection, the effect of fluorocitrate is largely reversed as Aldh1/1- and GFAP-positive astrocytes are again present at the injection site. Blue: DAPI, Green: GFAP, Red: Aldh111. Scale bar: $500 \mu \mathrm{m}$. 
2. The presented data on the role of astrocytes for neuronal functions in the peripheral ME-model are also relevant in the context of direct injuries of the neocortex. The authors should add a paragraph to discuss their own findings of astrocyte mediated neuronal hyperactivity after ME with respect to functional reorganization processes after stroke and/or traumatic brain injury.

We would like to thank reviewer 1 for this nice suggestion. Findings about cortical plasticity induced by the ME-model can indeed be relevant in the case of stroke and traumatic brain injury ( $\mathrm{TBI}$ ) as astrocytes have been shown to be involved in the functional recovery processes after brain trauma. We have included a paragraph in the discussion, right before our conclusion, in which we explain how our results are related to findings in stroke and $\mathrm{TBI}$ and also how our $\mathrm{G}_{\mathrm{i}}$ GPCR astrocyte activation approach could also be beneficial for boosting neuronal recovery in these conditions.

(Line 686 - 693)

Reviewer: 2

Comments to the Author

This manuscript reports upregulation in astrocytic density upon visual deprivation in the deprived visual cortex. "Metabolically silencing" astrocytes led to a decreased neuronal reactivation and recovery. Similarly, DREADD activation with Gi (and not of Gq) GPCR of astrocytes led to an amplification of cortical plasticity and recovery.

While I appreciate the idea and background of the study, there are a number of issues with the work.

1. The difference between $G_{i}$ and $G_{q}$ GPCR activation is unexpected, yet interesting and calls for further experiments. The authors hypothesize the involvement of possible downstream signaling pathways, which they should pharmacologically probe to make this more than just an idea.

We would like to thank reviewer 2 for the thorough analysis of our manuscript and for providing us with valid comments. We fully agree that the difference between $G_{i}$ and $G_{q}$ GPCR activation in the context of ME-plasticity is intriguing and already considered to attempt to pharmacologically phenocopy the Gi-GPCR outcome. However, revealing how these differences arise in the ME model turned out near impossible for several reasons. Even though we mention in the manuscript which pharmacological targets are of interest based on previous research (see also below for related preliminary data), we still remain quite uninformed about what pharmacological validation to perform, and in addition are also faced with a number of technical limitations specifically arising from chronic pharmacological manipulation (7-week time frame).

Technical limitations: Astrocytes and neurons possess many of the same receptors for which we lack drugs to specifically target the astrocytes, potentially leading to off-target effects. Our DREADD approach leads to very local effects whereas a pharmacological 
strategy would induce brain-wide effects. Considering that the neuronal reactivation in the ME model is partly mediated by cross-modal inputs, a brain-wide approach could therefore also impact neuronal activity in the regions surrounding the visual cortex, making interpretation of the results very complex. Another issue that complicates using pharmacology in the ME model is the duration of the recovery period. As the drugs would have to be given for 7 weeks, there is a high chance of inducing desensitization of the target receptor, which would again prevent us from correctly analyzing our data.

\section{Preliminary data:}

We already attempted to pharmacologically phenocopy our DREADD effect on neuronal recovery. As previously described in Durkee et al., $\mathrm{G}_{i}$ and $\mathrm{G}_{\mathrm{q}} \mathrm{GCPR}$ activation was shown to induce glutamate release from hippocampal astrocytes in acute slices (Durkee, 2019). We thus hypothesized that $G_{i}$ and $G_{q}$ GPCR activation of cortical astrocytes might be influencing plasticity processes by increasing extracellular glutamate levels. We therefore performed a chronic (7 weeks) pharmacological manipulation of the astrocyte-specific glutamate reuptake transporter GLT-1 through systemic delivery of dihydrokainate (DHK) antagonist with Alzet osmotic minipumps in ME mice. We have included the typical line graph analysis of the zif268-expression levels of DHK and vehicle (PBS) 7wME mice in which no statistically significant drug effect could be observed (Revision Figure 1). We decided not to include the DHK data in the manuscript since the lack of an effect might be attributed to a number of technical factors as explained above.
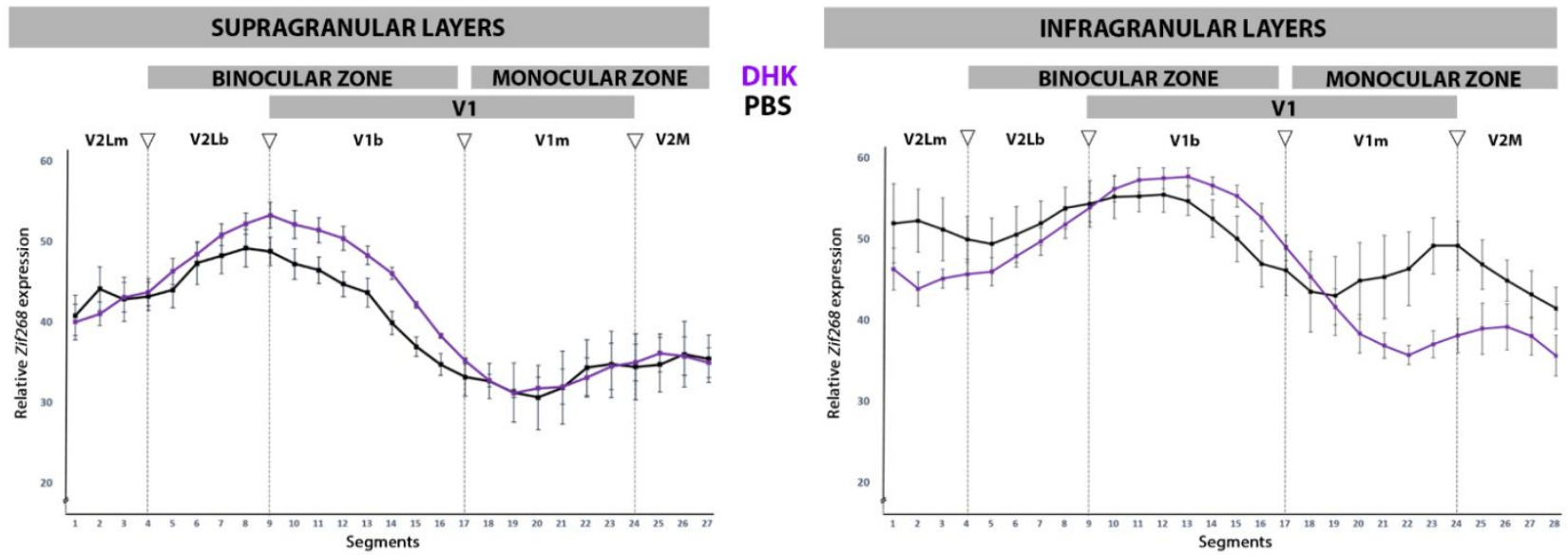

Revision Figure 1. Long-term systemic delivery of dihyrokainate (DHK) does not influence neuronal recovery after monocular enucleation. Line graph representations of the relative zif268 expression levels measured as the averaged OD value per segment from lateral to medial for $7 \mathrm{wME}$ that received long-term systemic delivery of PBS (black) and DHK (purple) through osmotic minipumps. Zif268 expression levels in the supragranular layers (II/III) and IV (left) and the infragranular layers $\mathrm{V}$ and VI (right) are displayed for the monocular (V1m, V2M) and binocular zone (V2Lb, V1b) of the visual cortex. Error bar represent SEM. PBS: $n=4$, DHK: $n=5$.

In our discussion we also hypothesize about the possibility of reverting astrocytes, through $\mathrm{G}_{i}$ signaling, towards a more synaptogenic phenotype capable of promoting 
plasticity. As an example we mention that in striatal astrocytes $G_{i}$ GPCR activation has been found to promote the release of thrombospondin-1 (TSP1), capable of enhancing excitatory synapse formation (Nagai, 2019). However, this study was focused on striatal astrocytes, which are anatomically, molecularly and functionally different from cortical astrocytes (Matias, 2019). Gi GPCR manipulation has also been shown to elicit a different response in astrocytes of different brain regions (Chai, 2017). Even though we thus hypothesize that $G_{i}$ GPCR activated cortical astrocytes become more synaptogenic, it may well be through another molecular strategy.

If we would still attempt to pharmacologically test the TSP1 involvement, we would choose to counteract the DREADD-induced TSP1 release with gabapentin (TSP1 receptor blocker) injections. Based on gabapentin's half-life (6 hours) we would have to administer the drug on a daily basis during the full 7 week recovery period (Kim, 2016). However, Yang (2016) showed that insensitivity to gabapentin starts from the second week of administration. In addition, gabapentin also affects GABA and glutamate levels in the cortex, thereby influencing plasticity, again preventing us from making any conclusions about the mode of action of Gi DREADD on the plasticity outcome (Cai, 2012; Cunningham, 2004).

In conclusion, based on the existing information and pharmacological tools we are not capable of elucidating $G_{i}$ and $G_{q}$ GPCR differences in the ME model, at this time and thus apologize to the reviewer for not being able to provide him with the requested information and hope our explanation as to why is satisfying.

2. Methods: After washing with PBS, the sections were blocked for 60 min with normal goat serum (Merck Millipore, Overijse, Belgium) and overnight incubated with the primary antibodies diluted in Tris-NaCl blocking buffer (TNB). Please add the temperature at which the sections were incubated.

Thank you for pointing this out, the sections were incubated at room temperature. We have now corrected this in the manuscript. (Line 240)

\section{Figures 2B: Unclear which one is ME and which one ctrl.}

As figure 2B was only intended to indicate that our Aldh1l1 immunostaining is clearly labeling protoplasmic astrocytes, based on their typical star-shaped morphology, both pictures were of control animals. We appreciate the comment/suggestion and have adapted this figure to show one control (top picture) and one ME (bottom picture) example of Aldh1l1+ astrocytes. We adapted the figure legend accordingly. 
a

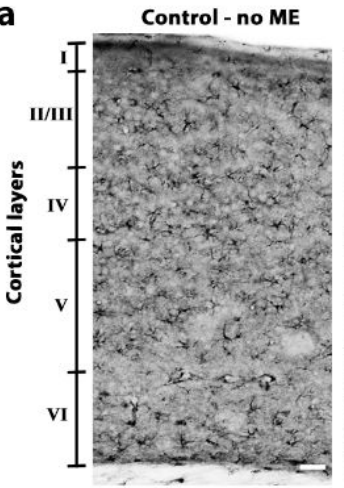

C

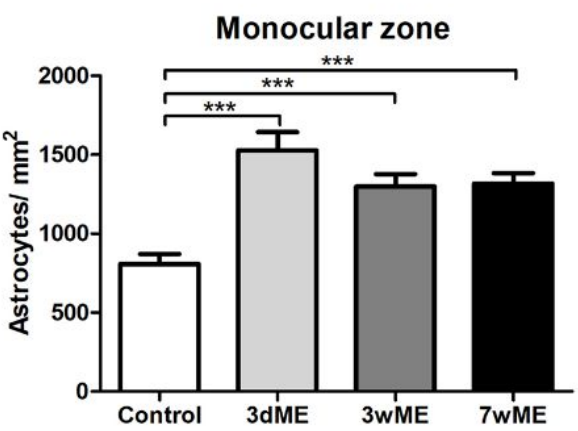

e

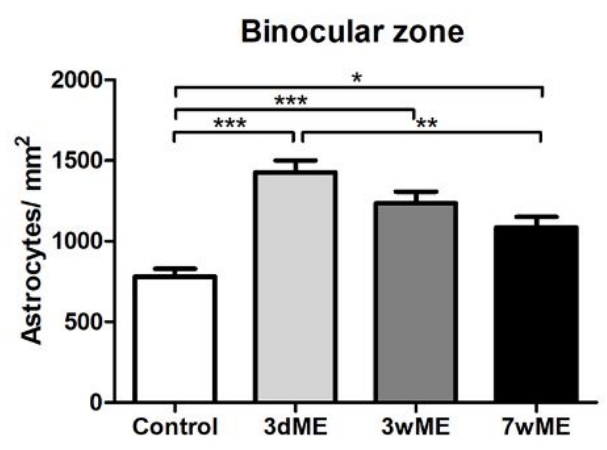

b

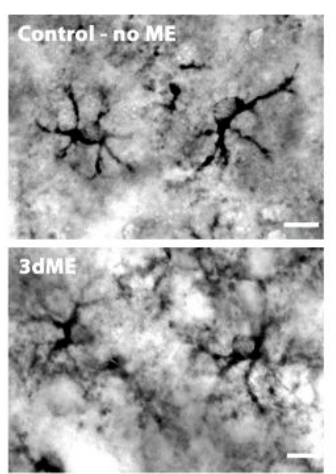

d

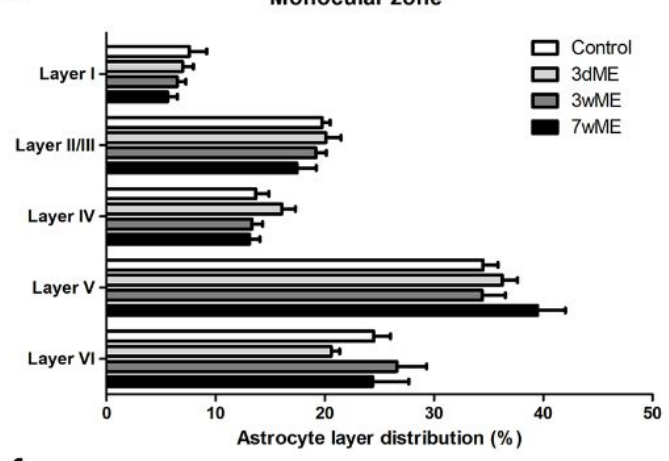

f

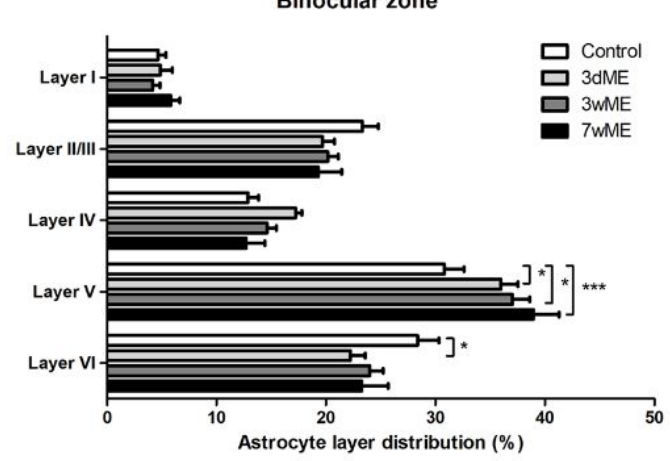

Figure 2. Time-dependent effect of monocular enucleation on the density and layer distribution pattern of Aldh1/1-positive astrocytes in the monocular and the binocular zone of the deprived visual cortex.

a. Representative images showing Aldh1/1-positive astrocytes across all layers of the visual cortex in control normal sighted (left) and 3-day enucleated mice (3dME) (right). Scale bar: $50 \mu \mathrm{m}$. b. High magnification picture showing clear protoplasmic astrocyte labeling based on typical star-shaped cellular morphology in control normal sighted (top picture) and 3dME mice (bottom picture). Scale bar: $10 \mu \mathrm{m}$. c. Astrocyte density in the monocular zone at different times post-enucleation represented as astrocytes $/ \mathrm{mm} 2$. One-way ANOVA, Bonferroni post hoc tests, ${ }^{* * *} p$-value $<0,001$. d. Astrocyte layer distribution pattern in the monocular zone for all analyzed conditions. Two-way ANOVA, Bonferroni post hoc tests. e. Astrocyte density in the binocular zone at different times post-enucleation represented as astrocytes $/ \mathrm{mm} 2$. One-way ANOVA, Bonferroni post hoc tests, ${ }^{*} p$-value $<0,05 ;{ }^{* *} p$-value $<0,01 ;{ }^{* * *} p$-value $<0,001$. f. Astrocyte layer distribution pattern in the binocular zone for all analyzed conditions. Two-way ANOVA, Bonferroni post hoc tests, * $p$-value $<0,05$; ${ }^{* * *} p$-value $<0,001$. ( $n=6$ for all conditions). $3 \mathrm{dME}, 3$ days post-enucleation; $3 \mathrm{wME}, 3$ weeks post-enucleation; $7 \mathrm{wME}, 7$ weeks post-enucleation. 
4. "Metabolically silencing" astrocytes with the injections of fluorocitrate is not a well-established approach and seems unspecific. The authors need to take a step back with the interpretation of these data. There can be countless effects of this intervention.

We understand the reviewer's concern that using a gliotoxin to silence astrocytes might be unspecific. Several recent publications exist in which the fluorocitrate approach was also used for in vivo silencing of astrocytes (Ishihara, 2019; Sobrinho, 2017; Wang, 2018; Zhang, 2019). We are not aware of any other/better method currently available that can be implemented to acutely induce a widespread inhibition of astrocytes in vivo.

The specificity of fluorocitrate for astrocytes stems from its preferential uptake into astrocytes, similar to exogenous citrate, where it inhibits aconitase and thereby blocks the TCA cycle (Fonnum, 1997; Hassel, 1992; Paulsen, 1988; Westergaard, 1994). In the context of too high a dose of fluorocitrate, the possibility exists that it could be taken up by other cells leading to off target effects. We therefore used existing literature to choose an optimal dose that elicits inhibitory effects only on astrocytes, and did tests with different concentrations in order to obtain a concentration that induces an extensive silencing of astrocytes whilst not affecting surrounding neuronal and glial cells, as illustrated in figure 3 of the original manuscript.

Prolonged administration can also lead to off target effects: chronic fluorocitrate infusion will lead to astrocyte death and, as a consequence, reactive microglia are observed surrounding the injection site (Kuter, 2018). Even though our data as well as current literature shows fluorocitrate to be highly specific for astrocytes, we acknowledge the possibility that at a later time point, as a secondary response, microglia will be affected. In sum, our fluorocitrate manipulation consisted of one single injection and did not induce a high degree of cell death (Revision Figure 2) reducing the chance for such secondary effects substantially.

We still understand the issue of the reviewer and have adapted the text in order to clarify to the reader that other cell types might still be affected as a secondary response to the astrocytic silencing. (Line 586)

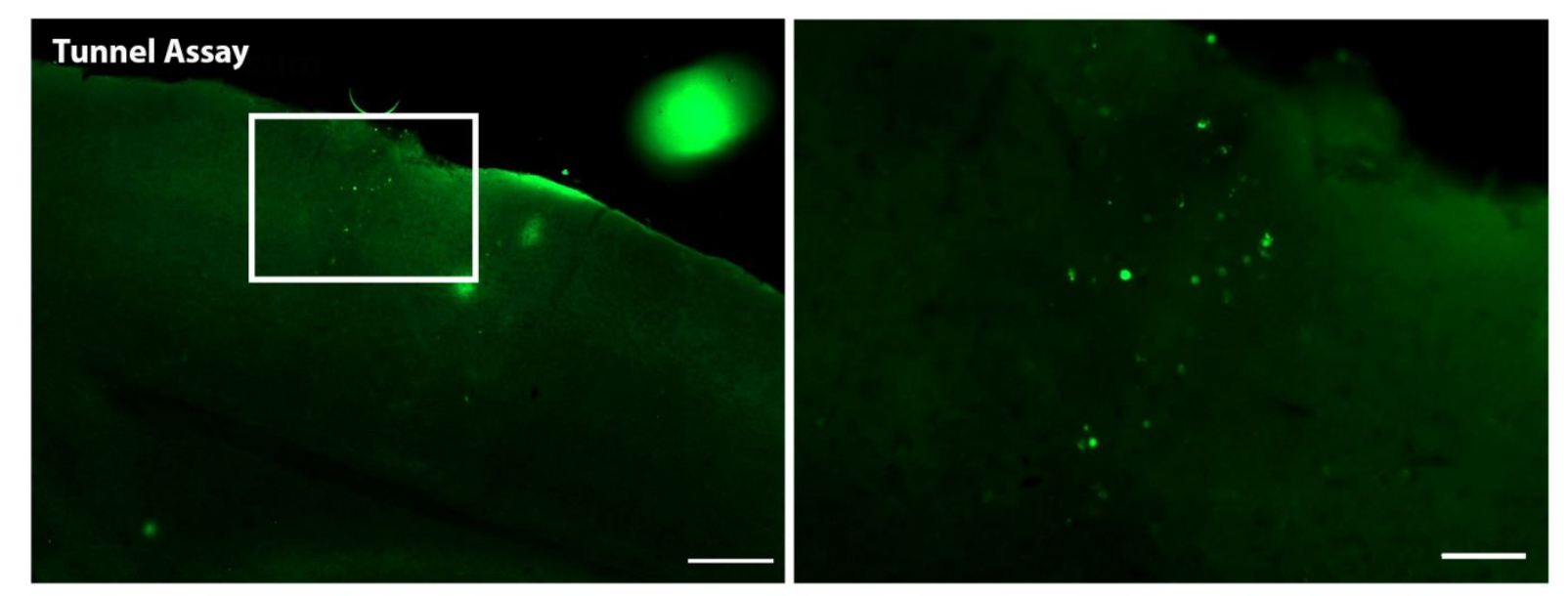

Revision Figure 2. A single fluorocitrate injection does not induce extensive cell death. Only a few apoptotic cells could be observed at the fluorocitrate injection site at one day post-injection. Green: Tunnel assay-labeled apoptotic cells. Scale bar: $200 \mu \mathrm{m}, 50 \mu \mathrm{m}$. 
5. TMEM119 is not really the optimal antibody to monitor changes in microglia. While Iba1 shows an increase in immunoreactivity after LPS injection, TMEM119 does not show gross changes (this is the initial paper characterizing the antibody https://www.pnas.org/content/113/12/E1738, Figure3D).

There a no ctrls shown for NeuN and microglia. It is therefore not really possible to claim that neurons and microglia are not affected after fluorocitrate injections.

The NeuN antibody was raised in mouse. It shows this increased background at the site of fluorocitrate, indicating that the secondary antibody recognizes some upregulated immunoproteins (possibly due to microgliosis?) in the tissue.

We agree with the reviewer that a TMEM119 staining will label the entire microglial population, making it difficult to separate reactive from non-reactive microglia, and that, in order to study microgliosis, IBA1 is the antigen target of choice.

The reason why we opted for TMEM119 is to observe if fluorocitrate induced an apparent effect on the microglial cell density comparable to what we observed for the Aldh1l1+ astrocyte population (please compare Figure 3a,b - red channel). We therefore chose to use a pan microglial marker as we did for the astrocytes with Aldh1l1. We reasoned TMEM119 to be a better choice than IBA1 since it specifically labels microglial cells and not blood-derived macrophages (Satoh, 2016). Considering that we deliver both fluorocitrate and saline through intracranial injections, the damaged tissue will most probably be infiltrated by macrophages preventing us from correctly interpreting differences in the relative number of microglia should we have used IBA1.

The reviewers concern about the anti-mouse NeuN antibody creating background due to immunoproteins is in our opinion also resulting from the damage induced by the stereotactic injections.

To respond to the reviewer's concern, we have performed an additional IBA1-staining (mouse antibody) on sections of 1-day post-injection, saline- (a) or fluorocitrate- (b) injected animals. We observed an intensive background labeling at the injection site in both conditions, preventing us from analyzing alterations in the microglia population. We also provide additional images from nearby the injection site where the IBA $1^{+}$microglia can be clearly distinguished, thereby showing that our protocol for IBA1 immunostaining is working (Revision Figure 3 ). In addition these images also show that IBA $1^{+}$microglia

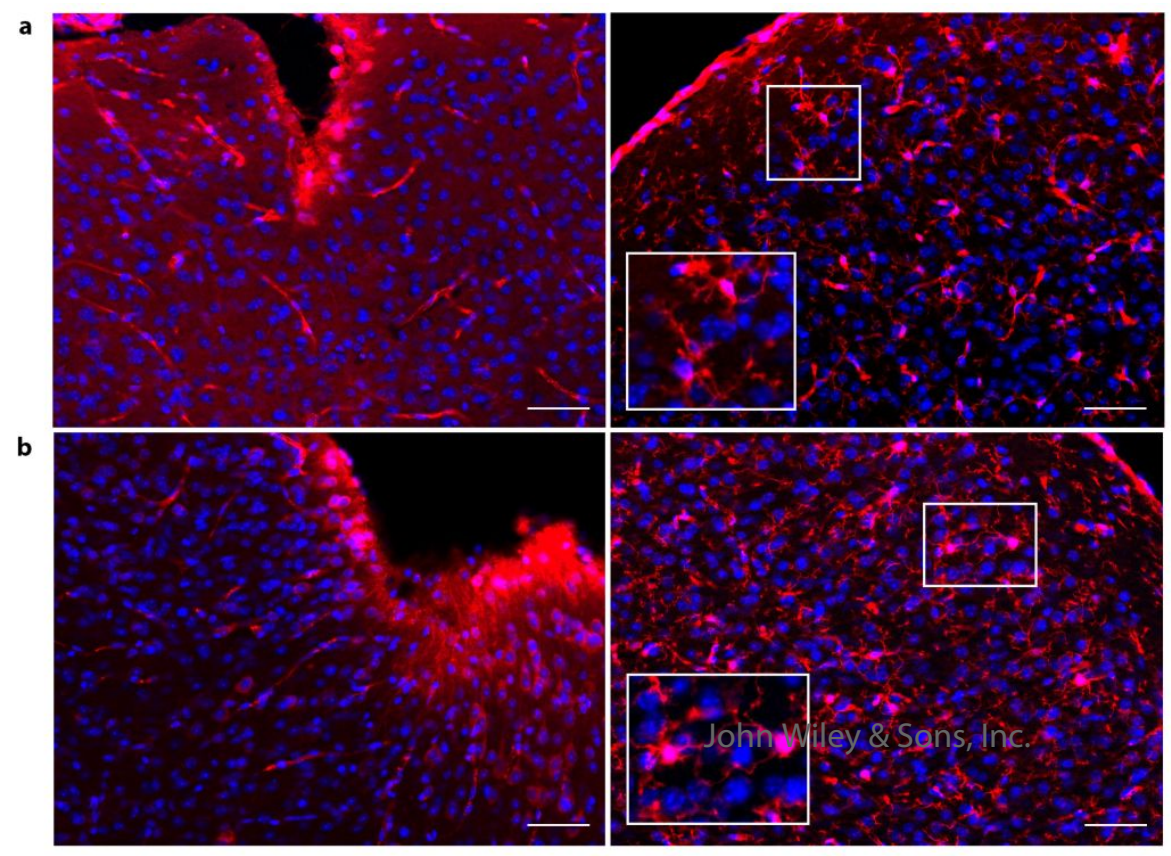
nearby the injection site have a ramified appearance instead of an amoeboid one, indicating that they are not reactive (Davis, 1994). 


\begin{abstract}
Revision Figure 3. IBA1 immunoreactvity in response to saline and fluorocitrate at one-day post injection.

No differences can be observed between IBA1 labeling of saline- (a) or fluorocitrate- (b) injected animals at (left panels) or nearby (right panels) the injection site on the same section. Blue: DAPI, Red: IBA1, Scale bar: $50 \mu \mathrm{m}$.
\end{abstract}

6. Figure 3: Please provide high resolution images to be able to judge the condition of the different cell types, comparable to the pictures in figure 5.

We prepared a new figure, including the requested high-resolution images, to allow the reviewer to judge the shape and relative number of the neurons and the microglia in both saline- and fluorocitrate-injected animals at one day post-injection(New Supplementary Figure 1). We are confident that from this figure it is clear that no apparent changes in the neuronal and microglial population can be observed between saline and fluorocitrateinjected mice. We have adapted the main body of the text in accordance. We added the new supplementary figure and its legend to the revised Supplement. (Line 586) 

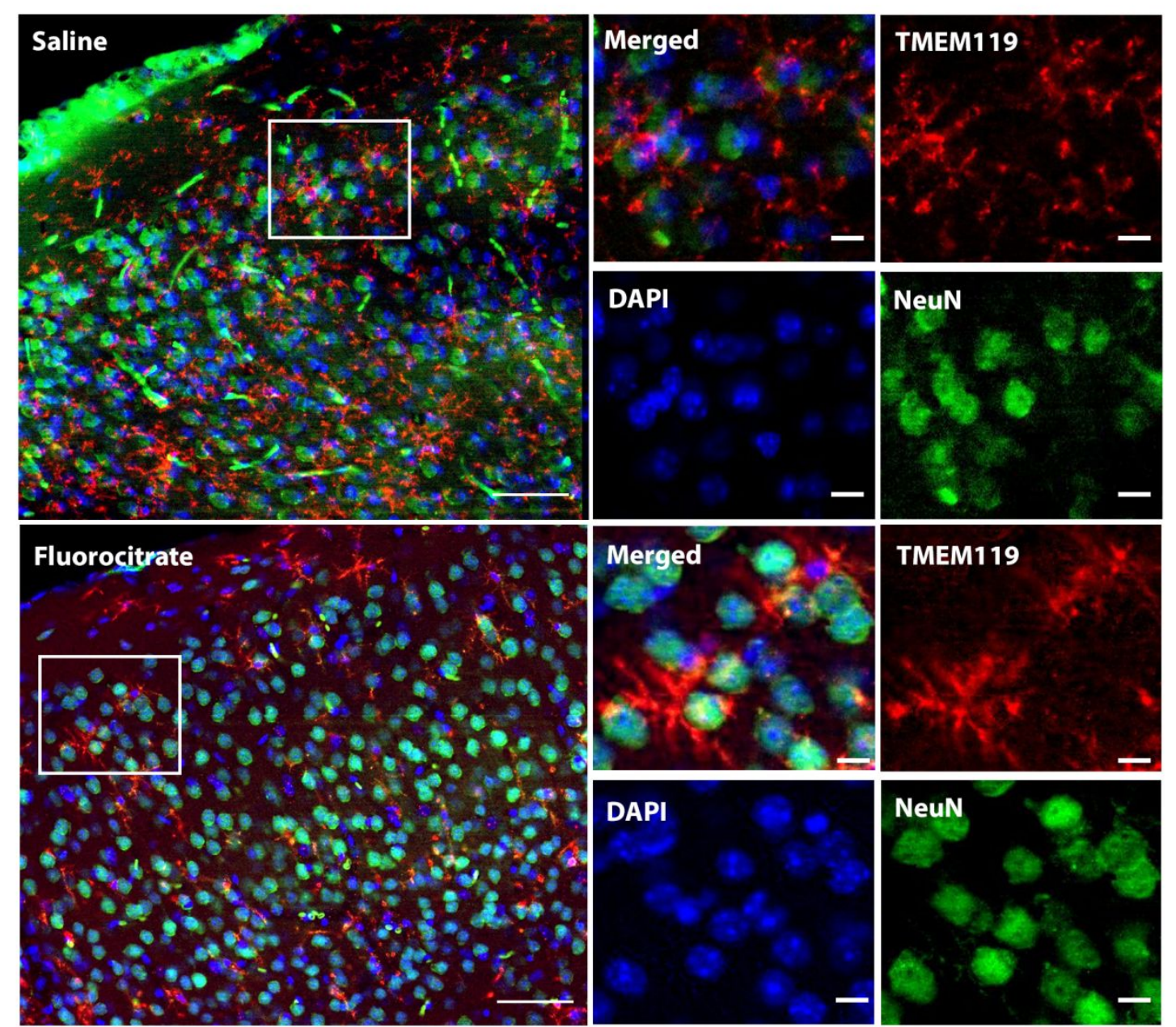

TMEM1 19
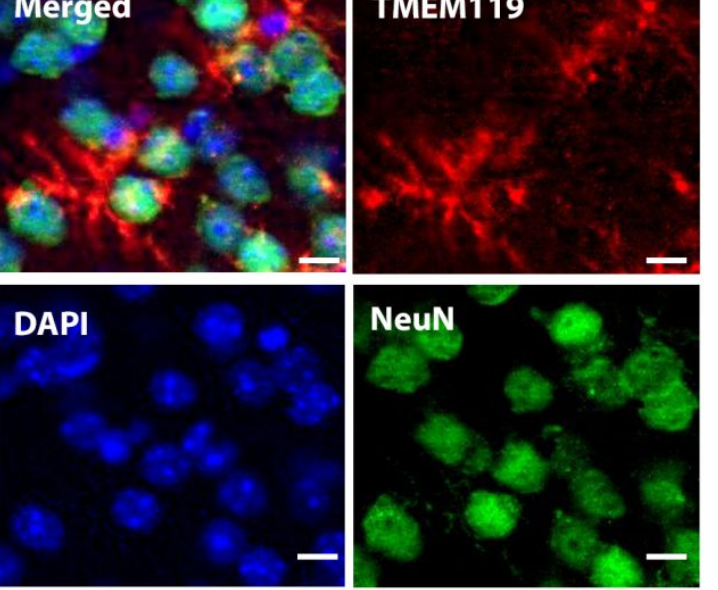

Supplementary figure 1. Neuronal and microglial cell are not affected by fluorocitrate at one day post-injection. No differences in neuronal or microglial cell shape or distribution can be observed one day post-injection between saline- (a) or fluorocitrate- (b) injected mice. Red: TMEM119, Blue: DAPI, Green: NeuN. Scale bar: $50 \mu \mathrm{m}, 10 \mu \mathrm{m}$

\section{References}

Cai, K., Nanga, R. P., Lamprou, L., Schinstine, C., Elliott, M., Hariharan, H., ... Epperson, C. N. (2012). The impact of gabapentin administration on brain GABA and glutamate concentrations: a $7 \mathrm{~T}^{1} \mathrm{H}-\mathrm{MRS}$ study. Neuropsychopharmacology: Official Publication of the American College of Neuropsychopharmacology, 37(13), 2764-2771. https://doi.org/10.1038/npp.2012.142

Chai, H., Diaz-Castro, B., Shigetomi, E., Monte, E., Octeau, J. C., Yu, X., ... Khakh, B. S. (2017). Neural Circuit-Specialized Astrocytes: Transcriptomic, Proteomic, Morphological, and Functional Evidence. Neuron, 95(3), 531-549.e9. https://doi.org/10.1016/j.neuron.2017.06.029

Cunningham, M. O., Woodhall, G. L., Thompson, S. E., Dooley, D. J., \& Jones, R. S. G. (2004). Dual effects of gabapentin and pregabalin on glutamate release at rat 
entorhinal synapses in vitro. European Journal of Neuroscience, 20(6), 1566-1576. https://doi.org/10.1111/j.1460-9568.2004.03625.x

Davis, E. J., Foster, T. D., \& Thomas, W. E. (1994). Cellular forms and functions of brain microglia. Brain Research Bulletin, 34(1), 73-78. https://doi.org/https://doi.org/10.1016/0361-9230(94)90189-9

Durkee, C. A., Covelo, A., Lines, J., Kofuji, P., Aguilar, J., \& Araque, A. (2019). Gi/o protein-coupled receptors inhibit neurons but activate astrocytes and stimulate gliotransmission. Glia, 67(6), 1076-1093. https://doi.org/10.1002/glia.23589

Fonnum, F., Johnsen, A., \& Hassel, B. (1997). Use of fluorocitrate and fluoroacetate in the study of brain metabolism. GLIA. https://doi.org/10.1002/(SICl)10981136(199709)21:1<106::AID-GLIA12>3.0.CO;2-W

Hassel, B., Paulsen, R. E., Johnsen, A., \& Fonnum, F. (1992). Selective inhibition of glial cell metabolism in vivo by fluorocitrate. Brain Research, 576(1), 120-124. https://doi.org/https://doi.org/10.1016/0006-8993(92)90616-H

Ishihara, Y., Itoh, K., Oguro, A., Chiba, Y., Ueno, M., Tsuji, M., ... Yamazaki, T. (2019). Neuroprotective activation of astrocytes by methylmercury exposure in the inferior colliculus. Scientific Reports, 9(1), 13899. https://doi.org/10.1038/s41598-01950377-9

Kim, S. K., Hayashi, H., Ishikawa, T., Shibata, K., Shigetomi, E., Shinozaki, Y., ... Nabekura, J. (2016). Cortical astrocytes rewire somatosensory cortical circuits for peripheral neuropathic pain. The Journal of Clinical Investigation, 126(5), 19831997. https://doi.org/10.1172/JCl82859

Kuter, K., Olech, Ł., \& Głowacka, U. (2018). Prolonged Dysfunction of Astrocytes and Activation of Microglia Accelerate Degeneration of Dopaminergic Neurons in the Rat Substantia Nigra and Block Compensation of Early Motor Dysfunction Induced by 6-OHDA. Molecular Neurobiology, 55(4), 3049-3066.

https://doi.org/10.1007/s12035-017-0529-z

Matias, I., Morgado, J., \& Gomes, F. C. A. (2019). Astrocyte Heterogeneity: Impact to Brain Aging and Disease . Frontiers in Aging Neuroscience , Vol. 11, p. 59. Retrieved from https://www.frontiersin.org/article/10.3389/fnagi.2019.00059

Nagai, J., Rajbhandari, A. K., Gangwani, M. R., Hachisuka, A., Coppola, G., Masmanidis, S. C., ... Khakh, B. S. (2019). Hyperactivity with Disrupted Attention by Activation of an Astrocyte Synaptogenic Cue. Cell, 177(5), 1280-1292.e20. https://doi.org/https://doi.org/10.1016/j.cell.2019.03.019

Paulsen, R. E., Contestabile, A., Villani, L., \& Fonnum, F. (1988). The effect of fluorocitrate on transmitter amino acid release from rat striatal slices. Neurochemical Research, 13(7), 637-641. https://doi.org/10.1007/BF00973281

Satoh, J., Kino, Y., Asahina, N., Takitani, M., Miyoshi, J., Ishida, T., \& Saito, Y. (2016). TMEM119 marks a subset of microglia in the human brain. Neuropathology, 36(1), 39-49. https://doi.org/10.1111/neup.12235 
Sobrinho, C. R., Gonçalves, C. M., Takakura, A. C., Mulkey, D. K., \& Moreira, T. S. (2017). Fluorocitrate-mediated depolarization of astrocytes in the retrotrapezoid nucleus stimulates breathing. Journal of Neurophysiology, 118(3), 1690-1697. https://doi.org/10.1152/jn.00032.2017

Wang, Y., Xie, L., Gao, C., Zhai, L., Zhang, N., \& Guo, L. (2018). Astrocytes activation contributes to the antidepressant-like effect of ketamine but not scopolamine. Pharmacology Biochemistry and Behavior, 170, 1-8. https://doi.org/https://doi.org/10.1016/j.pbb.2018.05.001

Westergaard, N., Sonnewald, U., Unsgård, G., Peng, L., Hertz, L., \& Schousboe, A. (1994). Uptake, Release, and Metabolism of Citrate in Neurons and Astrocytes in Primary Cultures. Journal of Neurochemistry, 62(5), 1727-1733. https://doi.org/10.1046/j.1471-4159.1994.62051727.x

Zhang, X., Shen, X., Dong, J., Liu, W.-C., Song, M., Sun, Y., ... Jin, X. (2019). Inhibition of Reactive Astrocytes with Fluorocitrate Ameliorates Learning and Memory Impairment Through Upregulating CRTC1 and Synaptophysin in Ischemic Stroke Rats. Cellular and Molecular Neurobiology, 39(8), 1151-1163. https://doi.org/10.1007/s10571-019-00709-0 